$3 \frac{1}{371.5}$ ir
635982 Grentzenberg, Max
1891

INVZ Die Spongien fauna der Ostsee.

1891 

Aus dem zoologischen Institut der Universität Kiel.

\author{
Die
}

\title{
Spongienfauna der Ostsee.
}

\section{Inaugtral-Dissertation}

/ zur Erlangung der Doktorwürde der phílosophịschen Fakultät

der

CHRISTIAN-ALBRECHTS-UNIVERSIT ÄT ZU/KYEL

/vorgelegt

von

Max Grentzenberg

aus Danzig.

Kiel 1891.

Druck yon Carl Bäckel. 
Rektoratsjahr 1890/91. Nr. 18.

Impi imatur : Krümmel, derz Dekan. 
Die ersten näheren Angaben über Schwämme in der Ostsee finden sich im ersten Jahresbericht der Kommission zur wissenschaftlichen Untersuchung der deutschen Meere in Kiel für das Jahr 1871. Es werden dort als in der Ostsee vorkommend sieben Species angegeben, die auf der Expedition zur physikalisch-chemischen und biologischen Untersuchung der Ostsee im Sommer $187 \mathrm{I}$ auf S. M. Avisodampfer "Pommerania "gedredgt und von E. Haeckel and $\mathrm{O}, \mathrm{Sch} \mathrm{mid}$ bestimmt wurden.

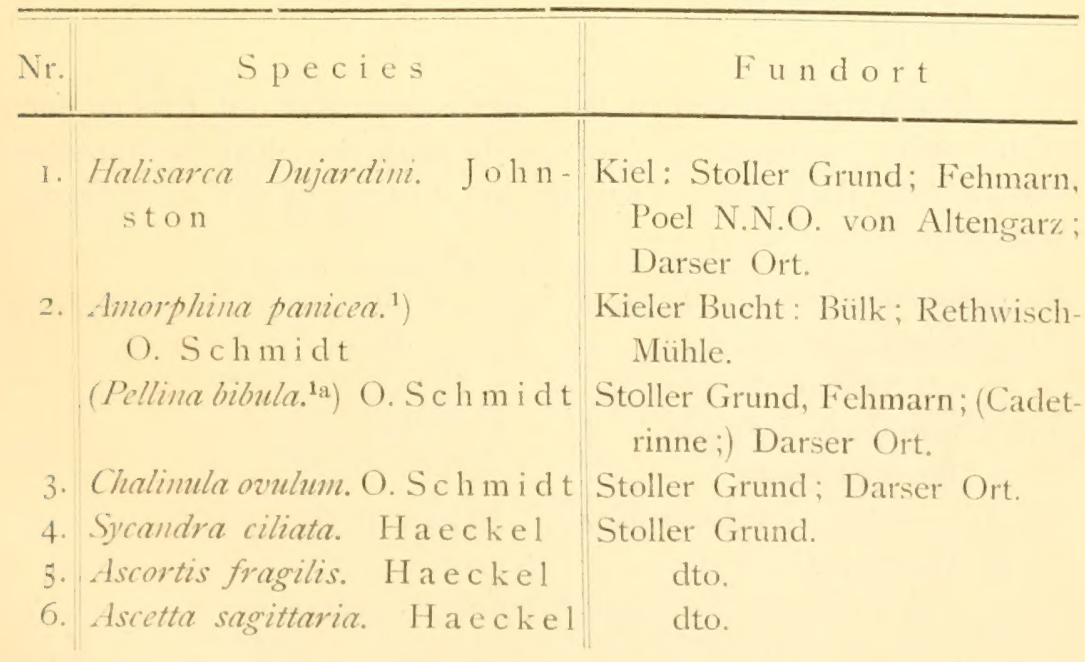

1) Richtiger Halichondria panicea, P a l I as, da das Genus Halichondria älter ist und daher beibehalten werden muss. Siehe S, II. identisch.

${ }^{1}$ i) Pellina bibula ist wie ich S, 13 zeigen werde mit Amorphina panicea 
Auf der zweiten Pommerania-Expedition im Sommer 1872 wurden für die Ostsee neu gefangen: ${ }^{2}$ )

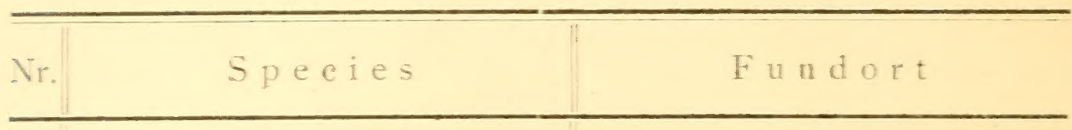

7. Polymastia mamillaris. B o- Gr. Belt.

werbank

8. Esperella (Esperia) lanugo. dto.

O. Sch midt

Beide wurden von $\mathrm{O}$. Schmidt bestimmt. Dazu gesellen sich die in den nächsten Jahren auf gelegentlichen Fahrten gesammelten und von Dahl bestimmten Arten :
9. IReniera aquaeductus.
Kieler Bucht.
O. Sch midt
10. Reniera (Isodyctia) varians. Gr. Belt.
B ow erban h
I. Chalina limbata. ${ }^{3}$ ) Bower- Kieler Bucht.
b a $\mathrm{nk}$

Durch Untersuchungen teils an frischem Material, teils an noch nicht näher bestimmtem Spiritus-Material aus dem zoologischen Museum in Kiel konnte ich für die Ostsee fernere vier Spezies feststellen:

12. Chalinula fertilis. Keller

13. Pachychalina rustica.

O. Sch midt

14. Lencosolenia coriacea.

B ow erban h

15. Lencosolenia (Ascetta) blanca. Kieler Bucht. Haechel

*) Jahresber. d. Kommission z. wissensch. Unters. d, deutschen Meere i, Kiel d. J. 1872 u, 1873. Serlin 1875 . S. I16 u. I18.

9) Diese Spongie wird bereits von L i e be r $k$ ï h $n$ unter dem Namen Spongia limbata Johnston als in der Wismarer Bucht vorkommend erwihnt. Arehiv für Physiologie 1859. S. 39S. 
Für die Kicler Bucht neu fand ich Reniera (Isodyctia) virians Bow erbank, eine Spongie, die man bisher in der Ostsee nur im grossen Belt gefunden hatte. Die Zahl der in der Ostsee vorkommenden Arten würde sich demnach auf I5 belaufen. Im folgenden habe ich sie in das System Vosmaer 's ${ }^{4}$ ) eingereiht, nur diejenigen Ordnungen, Klassen und Familien bericksichtigend, die Vertreter in der Ostsee haben.

\section{Porifera non calcarea.}

Ordo: Spiculispongiae.

Subordo: Oligosilicina.

Fam: Halisarcidae.

Gemus: Halisarca. D u j a r d in.

I)

Species: Hal. Dujardini. J o h n s to n.

Subordo: Clavulina.

Fam: Polymastidac.

Genus: Polymastia. B ow e r b a n k.

2)

Species: Pol. mamillaris. $\mathrm{B}$ o $\mathrm{w} \mathrm{e} \mathrm{r} b \mathrm{a} n \mathrm{k}$.

Ordo: Cornacuspongiac.

Subordo: Halichondrina.

Fam: Halichondridae.

Genus: Halichondria. F $1 \mathrm{e} \mathrm{m}$ ing.

3)

Species: Hal. panicea. P a 11 as.

Gemus: Reniera. O. S ch m id t.

4)

Species: Ren. aquaeductus. O. S c h m i d t.

5)

Ren. (Isodyctia) varians. B ow e $\mathrm{r}$ b a $n \mathrm{k}$.

Ge'mus: Chalimula. O. S c h mid t.

6)

Species: Chal. ovuhum. O. S ch m id t.

Chal. fertilis. K ell e r.

Gemus: Chalina. B ow e r b a n k.

8)

Species: Chalina limbata. B ow e r b a $n k$.

Gemis: Pachychalina. O. $\mathrm{S} \mathrm{chm} \mathrm{id} \mathrm{t.}$

9)

Species: Pach. rustica. O. S chm id t.

1) Vosmaer, Porifera 1886. S. 251 . 
Familia: Desmacidonidae.

Genus: Esperella (Esperia). Es p e r.

I O)

Species: Esperella (Esperia) lanngo. O. Sc h m i d t.

\section{Porifera calcarea.}

Ordo: Homocoela.

Familia: Asconidae.

Gemis: Lencosolenia. B ow e r b a $11 \mathrm{k}$.

1 I)

1 2)

Species: Lenc. coriacea. B ow e r b a $11 \mathrm{k}$.

(Ascetta coriaced. H a e c k e l).

Species: Leuc. (Guancha) blanca. M i clucho-

M a c l a y.

(Ascetta blanca. H a e c k e l).

Species: Lenc. (Ascetta) sagittaria. H a c ckel.

Species: Lenc. botryoides. J a mes-Cla rk.

(Ascortis fragilis. $\mathrm{H}$ a e c k e l).

(Grantia Lieberkïhnii. O. Sch $\mathrm{m}$ i $\mathrm{d} \mathrm{t}$ ).

Ordo: Heterocoela.

Familia: Syconidae.

Gemus: Sycon. R isso.

Spcries: Sycon ciliatum. Lice berk ii hn

Sycandra ciliata. H a e c k el.

Was die Verbreitung der Spongien in der Ostsee anbetrifft, so verdient die interessante Thatsache hervorgehoben zu werden, dass Spongien nur im westlichen Teile der Ostsee bis Rügen gefunden worden sind. In den vorher erwähnten Kommissionsberichten finden sich keine Angaben darüber, dass östlich von Rügen Spongien gefangen worden sind. $\mathrm{Brau} \mathrm{n}^{5}$ ), der den westlichen Teil des finnischen Meerbusens faunistisch untersuchte, und seine besondere Aufmerksamkeit " auf Spongien richtete, hat keine Spur von ihnen finden können. Die von der Kommission zur Untersuchung der deutschen Meere ${ }^{6}$ ) vorgenommene Einteilung der Ost-

5) M. B raun. Physikalische u. biologische Untersuchungen im westlichen Teile des finnischen Meerbusens. Archiv für die Naturkunde Liv-, Esth- u. Kurlands. Serie II. Band X.

6) Jahresbericht d. Kommission z. wissensch. Untersuchung d, deutschen Meere fiir 187 x. S. IX. 
see in ein westliches bis. Rügen reichendes und in ein östliches Becken würde also auch in bezug auf die Spongien zutreffend sein, die ein den übrigen in der Ostsee vorkommenden Tieren analoges Verhalten zeigen. Der Grund für diese Erscheinung ist einerseits in dem Zusammenhang des westlichen Ostseebeckens mit der Nordsee, andererseits in dem geringen Salzgehalt des östlichen Beckens zu suchen. Aber auch in dem westlichen Teile ist eine stetige Abuahme der Arten von Westen nach Osten zu bemerken; während an Spongien in der Kieler Bucht vierzehn Arten vorkommen, finden sich in der Travemünder Bucht nur noch fün Arten.

Am weitesten verbreitet sind; Halichondria panicea, daneben Pellina bibula, welche Spongie jedoch, wie ich Seite 13 zeigen werde, schwer von Halichondria panicea zu trennen ist, und Halisarca Dujardini. Nicht nur kommen sie häufig in der Kieler Bucht vor, sondern auch nach den Untersuchungen von Le n $z$ in der Travemünder und nach denen von $\mathrm{Bra} u \mathrm{n}$ in der Wismarer Bucht. I, enz ${ }^{i}$ ) hat in der Travemünder Bucht ausserdem noch Chalimula ovulum gefunden, einen Schwamm, welcher nach den Angaben im ersten Kommissionsberichte in der Kieler Bucht und bei Darser Ort vorkommt. In der Kieler Bucht scheint er jedoch äusserst selten vorzukommen, demn trotz eifrigen Suchens habe ich keine Exemplare von Chalimula omum finden können. Durch die Güte des Herrn Dr. Lenz erhielt ich Schwämme, die von ihm in der Travemünder Bucht gesammelt waren, zur Untersuchung. Es sei mir gestattet, an dieser Stelle Herrn Dr. Lenz für sein Entgegenkommen meinen besten Dank auszusprechen. Die Sammlung enthielt die vorher erwähnten vier Arten und Chalina limbata. Die Wismarer Bucht ist von $\mathrm{Bra} \mathrm{u} \mathrm{n}^{8}$ ) untersucht; an Spongien hat er daselbst Halichondria panicea, Pellina bibula und Halisarca Dujardimi gefunden. Clualina limbata war, wie vorher erwähnt, bereits von $\mathrm{Lieberk}$ ï h $\mathrm{n}^{9}$ ) in der Wismarer Bucht beobachtet worden. Die Kalkschwämme scheinen nur im westlichsten Teile der Ostsee vorzukommen: in der Kieler Bucht und im grossen Belt, denn

7) Lenz, die wirbellosen Tiere der Travemuinder Bucht. Teil I. Anhang I. zu dem Kommissionsberichte I874 u. I875.

8) M. Braun, faunistische Untersuchungen der Bucht von Wismar. Archiv der Frde. fuir Naturgeschichte in Mecklenburg. Jahrgang 42. IS88.

4) Siehe Anmerkung 3. S. 4. 
weder $\mathrm{L}$ e $\mathrm{nz}$ noch $\mathrm{Br}$ a $\mathrm{u}$ haben in der Travemünder bezichungsweise Wismarer Bucht selbige gefunden.

Nebenstehende Tabelle soll eine Uebersicht uber die Fundorte der Schwämme in der Ostsee geben mit Hinzufügung ihres $\mathrm{Ver}^{-}$ breitungsgebietes ausserhalb derselben.

Die nebenstehende Tabelle zeigt unter anderem, dass Chalimula fortilis und Pachychalina mistica bis jetzt nur im Mittelmeer und in der Ostsee gefunden sind. Es ist sehr wahrscheinlich, dass diese beiden Spongien auch in der dazwischen liegenden Meeresstrecke - in dem Atlantischen Ocean und in der Nordsee - vorkommen. Dass sie dort bisher nicht constatirt sind, liegt möglicherweise nur daran, dass die betreffenden Meeresabschnitte verhältnismässig wenig auf ihre Spongienfauna untersucht sind. Dasselbe müssen wir annehmen bei Lencosolenia b'anca (Kanarische Inseln) und Chalimula orulum (Grönländische Küste). Auch bei Esperella lamugo und Lencosolenia sagittaria, dic bis jetzt nur in der Ostsee gefunden sind, liegt die Vermutung nahe, dass sie im Atlantischen Ocean und in der Nordsee verbreitet sind.

Interessant ist das gänzliche Fehlen der reinen Hornschwämme in der Ostsee. Man nimmt heute allgemein an, dass sich die Hornschwämme aus den Kieselhornschwämmen entwickelt haben, indem eine Reduction der Kieselausscheidung eintritt und dancben ${ }_{\star}$ eine stärkere Entwicklung der Hornsubstanz. Diese Processe sollen namentlich in wärmeren Meeren an Schwämmen sich vollziehen. Daher dort die grosse Verbreitung der Hornschwämme. Das gänzliche Fehlen derselben in der Ostsee und den nördlicheren Meeren würde diese Hypothese bestätigen.

Bei der Beschreibung der einzelnen Arten habe ich ein besonderes Gewicht gelegt auf die Verschiedenartigkeit der Kieselgebilde und ihre Anordnung; es gelang mir bei einzelnen Species cine Anzahl von Kieselgebilden nachzuweisen, die frühercn Untersuchern entgangen sind. Näher eingegangen bin ich auf das Studium des histologischen Baues von Hal. panicea, da mir infolge der weiten Verbreitung dieses Schwammes in der Kieler Bucht stets frisches Material zur Verfügung stand.

Bei Chalina limbata und Esperella (Esperia) lamigo habe ich der Entstehung der Sponginsubstanz aus den sog. Spongoblasten eine besondere Aufmerksamkeit gewidmet. 


\begin{tabular}{c|c} 
S Pecics & $\begin{array}{c}\text { Fundort } \\
\text { in der Ostsec }\end{array}$ \\
\hline
\end{tabular}

1. Malisarea Duijurdini Johnston

2. Polpmastia mamillerris bower ba n k

3. Halichomatriar pernicery I'all as

4. Remiera aquateductus $\mathrm{Schmidt}$

5. Remiera (Lodyctior) a'derigns Buwerbank

6. Charlimbla ormbum Schmidt

7. Chalina limbata Bow erbank

8. Chalinea fertilis Keller

9. Pachyehalina rustica Schmidt

10. Esperella (Lisperia) lamugo Sch midt

11. Letuc. botryoides JamesClark (Gratia Lieberkiuhnii Schmidt) (Ascortis frorgilis Hacchel)

12. Lencosolenia coriacen Kicler Buclut Bow e r bank

\section{(Ascetta corviciea $\mathrm{Hackcl}$}

13. Lemesolemia (Ginanchar) blemea $\mathrm{Micl}$ ucho. Ma clay (. Ascetter blerned Hacckel)

I4. Lencosolemia (.Iscetta) 'Kicl, B., Gr. Belt sagitt. Ha e ckel

15. Sjecon ciliatum L. icberkiihn (Sircarmdra ciliater Hacekel
Kicl., Travemuind. Mittelnecr, Ntit.

Vismarer Bucht Occan, Nordice

(ir. Belt

Barents Meer, Brit. Kiiste, K.v. Norw.

Kicl., Travemünd, Mittelm., Atlant.

Wismarer Bucht | Ocean, Nordsce Kieler Buclit

Mittelneer, Nord$\mathrm{sec}$

Kicl. B., Gr. Belt Britiscle Kiiste Kiel., Travemund. Grönländ. Küiste B., Jarser Ort Kiel., Travemind., Vismarer Buclit

Kieler Bucht

Mittelmecr:Neapel, clto.

Gi. Belt

Kiel, B., Gi. Belt

Nord-atlantischer

Ocean, Küiste

von Norwegen

Küste von Frankreich

Britannicn und

Norwesen

ito.

Canarische Inscln,

Kiiste von

Brasilicn

Kiel, B,, Gr. Belt Atlantisch. Occan, 


\section{Porifera non calcarea. ${ }^{10}$ )}

Skclet schr sclten fehlend, sonst aus Kieselspikula, welche durch Spongin resp. Kiesel zusammengehalten sein können; oder aus Sponginfasem allein bestehend. Kanalsystem nach dem dritten oder vierten, nicmals nach dem ersten oder zweiten 'Typus gebaut.

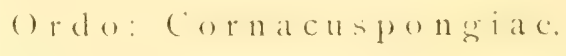

Shelet besteht cntweder aus, vorwiegend, monaxilen Spulula, welche durch mehr oder weniger Spongin zusammengekittet sind, oder nur aus Spongin mit oder ohne Verstärkung von fremelkörpern. Leben in Sec- brakischen und Süss-Wasser, meist nicht schr ticf.

$$
\text { Suburdo: Halichondrina. }
$$

Das Skclet wird hauptsächlich aus Spikula gebildet. Dic Quantität Spongin ist eft verschwindend gering. Ausser stabformigen Skeletnadeln oft sog. Fleischnadeln. Kanalsystem vorwiegend nach dem dritten Typus.

Familia: Halichondridac.

Nadeln in Zügen oder wirr durcheinander; selten ein Netzwerk von kubischen Maschen bildend. Spongin oft kaum vorhanden, oft stark entwickelt.

\section{Gomes: Halichondria. Fil e ming,}

Locker, trocken, sehr zerbrechlich. Die Nadcln, in Zügen oder wirr, sind Modifikationen von ac ${ }^{2}$ und $\mathrm{tr}^{2}$, meist schlank. Atlantischer Ocean, Mittelmeer. Untief.

10) Die Merkmale der Ordnungen, Unterordnungen und Familien sind Vos. maer's l'oriferi entuommen. 


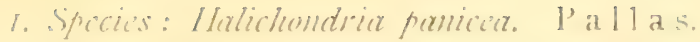

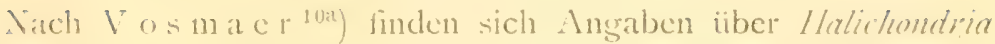

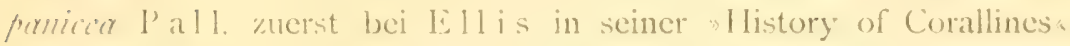
1755. der ihn Spongia medullam panis referens oder Crumb of Bread Sponge nannte. P'allas belegte ihn I 766 mit dem Namen Spengige panicere, und Johnston brachte ihn bei dem von Ficm ing i $\$ 28$ aufgestellten Gimus: Helichondria unter. Dic späteren englischen Autoren haben dicson Namen beibchalten.

In seincm Werke Grundzige einer Spongienfauna des atlantiscisen Gebietes: stellt $S \mathrm{ch}$ midt als neue Gattung Anorplina auf und charaliterisirt sic folgendermassen: „Kürzere oder längere umspitzige Nadehn in groben unregelmässigen Zügen oder gan\% wirr durcheinander. Keine Oberhaut oder nur stellenweisc." Von schon bekannten Arten reihte or in scin ncues Genus Herlichondrior prmicer P'all. cin. Da der Genusname Halichondria aber ein vicl älterer ist, so behalte ich ihn bei.

Ifalichondria faniced P'all. (1'ig. 1 11.2) ist ein Schwamm von der verschiedenartigsten Gestalt und meistens inkrusticrend auf den Algen Firstigaria, Firtellaria. Phyllophora und fincus; es will mir scheinen, als ob seine (iestalt in excwissem Nasse bedingt würde durch dic von ihm umwachsene Alge. Inkrustict er \%. B. auf Finrellaria. deren Zweige duinn und rund sind, so nimmt er cine rundliche und lïngliche Gestalt an. Anders wird seine Gestalt, wenn er auf Algen von blattförmigem Thallus inkrusticrt. Doch glaube ich, class dieser Einfluss nur in der Jugendzeit des Schwammes stattfinclet, wo die Pflanze ihm als Stuitze dient. Später entsendet der inkrusticrende T'eil Ausliufer und Seitenzweige, sodass der Schwamm eine unregrelmässige Gestalt crhält. Die äusserste Modifikation zeiggt or in einer $10-15 \mathrm{~cm}$ hohen Staude, deren einzelne Zureige cinen Durchmesser von 2-10 mm haben. Auch Bow erbank ${ }^{11}$ ) beschreibt solche fiomen von Ferlichondria pernicea, und or weist auf dic Achnlichlieiten in der Gestalt mit Polymastic marimillaris hin. Halichondria panien von dieser Gestalt fundet sich zwischen Fehmarn und Langeland und mir liegt ein lixemplar von dort vor.

\footnotetext{
1": Iosmacr, J'orilera r\$\$6. S. 337.

. li u
} 
Die Grösse des Sehrammes ist sehr verschieden; in eler Kieler Bucht schwankt sic zwischen 2 und $10 \mathrm{~cm}$.

libense wic dic Gestalt variint, so variirt auch dic liarbe. Vorluerschend ist dic gelbliche liarbe in allen 'Tönen, namentlich ins rötliche äbergehend. Häufig finclet sich auch eine gröine lïrbung melu oler weniger dunkel. Nicht selten trifft man die gelbe und die grime Farbe auf demselben Exemplar an. Oskula können fehlen oder vorhanden scin, ersteres ist häufiger der Fall; sind sie vorhanden, so erreichen sie einen Durchmesser von cinem Millimeter und sind unregelmässig in Abständen von I $\mathrm{cm}$ uber die Oberfläcle zcristreut. In einzelnen Fallen habe ich beobachtet, dass dic Haut. dic den Schwamm umgeben kann, sich papillenfömig über das Oskulum erhebt. Nicht immer treffen wir bei Hal. parlicea cine deutlich ausgeprägte Oberhaut an. Sie kann auch ganz fehlen oder nur stellenweise vorhanden sein. In der Oberhaut finden sich zahlreiche l'oren von ovaler oder rundlicher Gestalt: ihre Grösse beträgt $0,05-0,07 \mathrm{~mm}$. Die Konsistenz der Oberhaut ist verschieden; bald ist sie fest, sodass man sic vom Innenparenchym ohne Schwierigkeit abzichen kann, bald liegt sie nur lose und in sich locker demselben auf, sodass bei Versuchen, sie freizulegen, sic zerfaillt. Schmi d $^{{ }^{3 *}}{ }^{*}$ legt auf das Vorhandensein oder Fehlen der Oberhaut grossen Wert, und in den Spongien des atiantischen Gebietes" stelit er ein neues Genus Pellina auf und sagt: 》Der schon bekannte Typus, von dem wir fur diesc Gattung ausgehen, ist dic venetianische Reniora semitubulos $\mathrm{Scl}$ midt, eine unter vielen Formenvarietäten auftretende Spongie mit Renierennadeln in unregelmässigen Zügen oder bunt durcheinander. Sic ist höchst zerbrechlich, und nur die zusammenhängende Oberhaut verleiht einigen Zusammenhalt. Da wir Esperien mit Oberhaut, Desmacidonarten mit Oberhaut kennen, da wir ferner bei Tedania bestimmt zusammengehörige Arten mit und ohne Oberhaut kennen, so liesse sich auch diesc Pellina noch unmittelbar bei Anorphini unterbringen. As ein weiteres Charakteristikum der Gattung Pelline fuihrt $S$ ch midt die Anordnung der Nadeln zu einem schönen vicl- 
reihigen Netze an. Nuf beide Merkmale ist aber nichts zu geben. Schon Keller ${ }^{33}$ ) fand an ein und demselben lixemplar von R'mera semitubulosa grössere Röhren mit Oberhaut (Charakter der Gattung l'Pllina), andere Stellen, an der Basis, aber ohne ()berhaut und mit parallel oder ganz wirr durcheinander liegenden Nadeln (Charakter der Gattung Amorphina) und endlich feine Acste mit Nadeln, die regelmässig zu dıei oder vierseitigen Maschen angeordnet sind (Charakter der Gattung Renierer). Achnlich habe ich Exemplare von Halichondria panicer gefunden, die stellenweiso eine Oberhaut besassen und cine netzförmige Anordnung der Nadeln zeigten. Dic Gattung Pellina ist daher von Ifalichondria schwer zu trennen und man thut gut, beide zu identifizieren. Dic zur Gattung Pellina gehörende Species Pellina bibula S c h m i d t von der mir ein Exemplar im Original vorliegt, zeigt ebenfalls so weniy Unterschicde von Hal. panicea. P' a I l, dass sic nicht von letzterer zu tremen ist. Ebenso schwicrig ist von Halichondria pranice die von S c h m i d t ${ }^{14}$ ) aufsestellte Species Anorphina paciscens aus der Nordsee $z u$ trennen, die er folgendermassen beschreibt: Kurze, nicht oder wenig verzweigte Aeste ron dor Dicke eines starton Federkiels und ziemlich ausgebildeter Oberhaut, wodurch die Trennung von Prllina misslich wird. Dic Nadeln sind vorherschend grobe Umspitzen, zum Teil in Zügen. Dazwischen sehr feine Nadeh. Die Konsistenz des Parenchyms ziemlich fest." Der cinzige Unter. schicel zwischen. Anorphina paciscens und Halichondria panicea wirde nach S clu $\mathrm{m}$ dt also darin bestehen, dass erstere ausser den grossen Umspitzen noch sehr feine Nadeln besitzt. Hishchondria fanice hat aber auch stets neben den gewöhnlichen Umspitzen noch feinere Nadeln, wenn sie auch nicht so regchässig und so zathlreich vorkommen, wic bei Amorphina paciscens.

Dic Nadeln, die an der (Oberfläche des Schwammes sich zahlreicher vorfinden als im Innern, durchsetzen bei Halichnndria pornirea stets polyserial in Zuigen die Oberhaut und das Parenchym. Die Züge kömnen bald ein schön ausgeprägtes Netz bilden, bald

13. Keller, Leber den Bau von Reniera simitubulusa, Sch mi dt. \%eitsch.

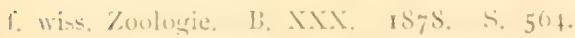

1t lalueshericht der Kommission zur wissensch. Lnters. d. detsch. Meere

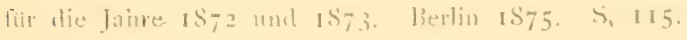


wirr durch einander verlaufen, ohne auch nur die Spur eines Netzes anzudeuten. An der ()berfläche ragen die Nadeln etwas hervor, sodass der Schwamm sich raul anfült. Mamnigfaltiger Art sind die Kieschnadeln, die ich bei Hal. panicea angetroffen habe. B ow e r b $\mathrm{n} \mathrm{k}^{15}$ ) zwar kennt bei diesem Schwamm nur zwei verschiedene Formen ron Nadeln, die er acerate und sub-fusiformiacerate nennt. Unter acerate spicula versteht er spicula of the same diameter for the great part of the length of the shaft, but decreasing equally near each termination and ending acutely at both; sub-fusiformi-acerate are spicula, having the greatest diameter at the middle of the shaft and decreasing gradually to each acute termination. Die acerate spicula sind lang und dick oder kurz und fein. Es sind dies jedoch bloss die Grundnadeln, die fuir den Schwamm charakteristisch sind. Ausser ihnen finden sich noch andere Nadelformen und in nebenstehender Tabelle habe ich dic bei IO Exemplaren gefundenen Nadeln aufgeführt.

Dic Bezeichnung der Nadeln habe ich F. F. S c h u l z e's und v. Le ndenfeld's Monographie Ueber die Bezcichnung der Spongicnnadeln", Berlin I 889 , entnommen. Es wäre 7.4 wünschen, dass diese Bezeichnungen bei allen Spongiologen Anerkennung fainden, damit endlich eine einheitliche Nomenklatur eingefuihrt wirde. Das Amphitorn und das Amphiox finden sich am haufigsten und zwar stets gewölbt, in vereinzelten Fällen geknickt; von verschiedener Länge und Dicke. Die ubrigen Nadeln treten nur vereinzelt auf; auch habe ich nicht beobachten könnnen, dass sie einem bestimmten Teile des Schwammes besonders angehören, wie ich ziberhaupt nicht einen Unterschied in der Nadelbildung bei den Nadeln der Haut, Dermalia, und den Nadeln des Parenchyms, Parenchymalia genannt, finden konnte. Interessant ist noch das Vorkommen von kreuzförmigen Kieselgebilden. Wir haben sie nacl den vortrefflichen Untersuchungen von F. E. Schulze $\mathrm{e}^{16}$ ) als Ruickbildungen anzuschen. Das Tylostyl ist bis jetzt erst bei den Suberitidac und Clavulina beobachtet worden. Das Oxydiactin

\footnotetext{
15) Ifow erbank, A Monograph of British Sipongiadae. Vol. I. p. 229).

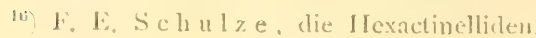




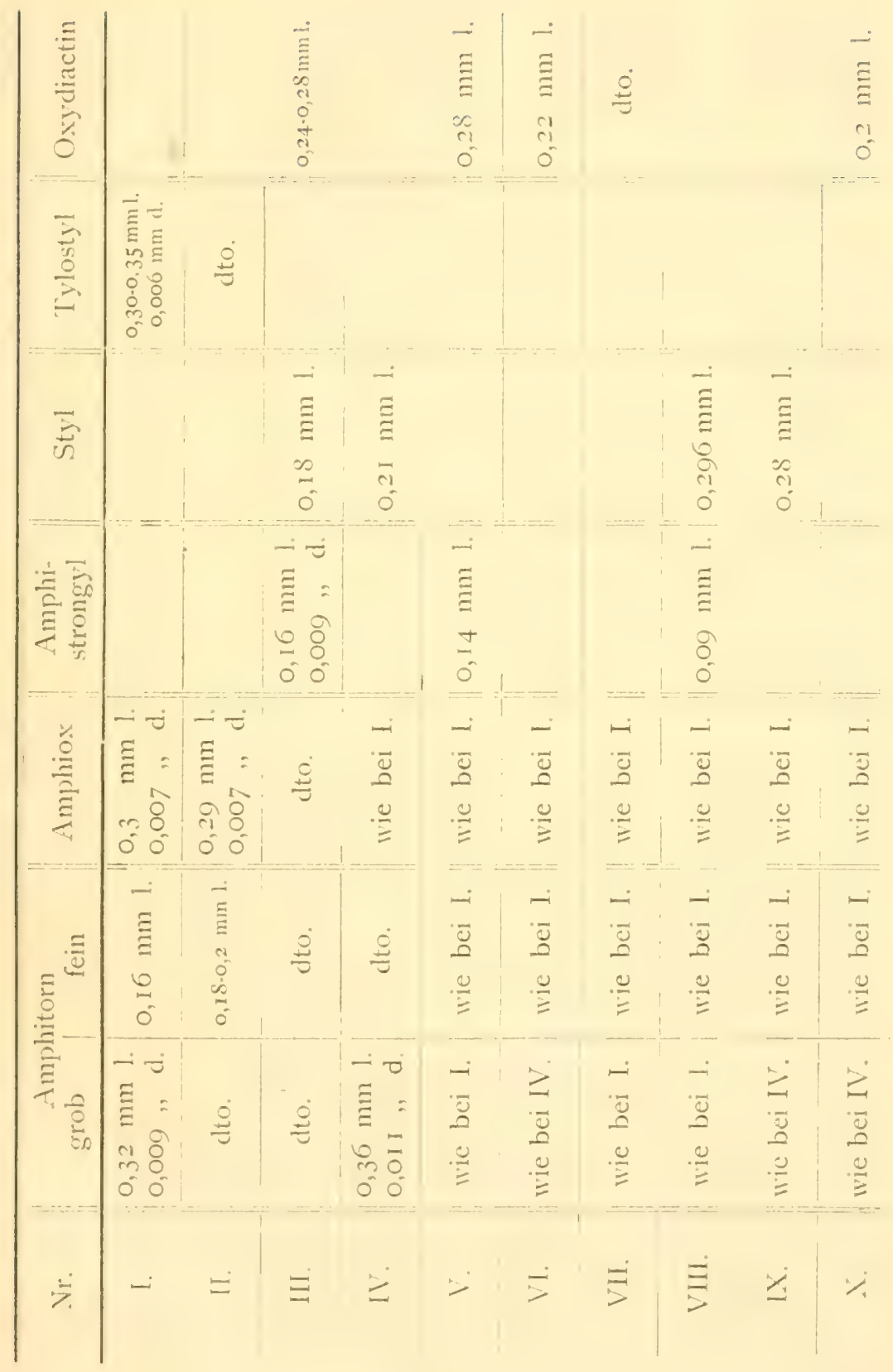


kommt nach $\mathrm{O}$. S $\mathrm{Shm} \mathrm{idt}^{3}$ ) bei Anorplina senitrix vor. Monstrositaten in der Nadelbildung sind häufig; in Fig. $3 . g, h, i, k$ habe ich cinige abgebildet.

$\mathrm{K}$ a n a Is ys tem.

Das Kanalsystem von Hal. pranice ist sehr komplizirt gebaut. Nach V o s ma e ${ }^{1}$ ) kann man das Kanalsystem der Spongien unter 4 Typen bringen, die aber, wie er selbst bemerkt, nicht scharf von einander zu trennen sind. Das Kanalsystem von Hal. panicea wiirde dem vierten Typus zuzurechnen sein. Man unterscheidet bei allen Spongien ein zuführendes und ein abführendes System. Das zufuihrende System besteht bei Hal. panicea aus zweierlei Kanälen, aus kleineren und grösseren. Die kleineren stehen in Verbindung mit den Poren, verlaufen senkrecht zur Oberfläche und ziemlich parallel. Sie führen in grössere, longitudinal verlaufende Kanäle. Dadurch, dass sich sowohl oft mehrere kleinere Kanäle, bevor sie in die srösseren muinden, mit einander vereinigen, als auch durch die häufige Vereinigung der grösseren longitudinal verlaufenden Kanäle, bietet das Kanalsystem auf Quer- und Längsschnitten ein komplizirtes Aussehen. Die grösseren Kanäle stehen entweder direkt in Verbindung mit den Geisselkammern, oder aber es führen kleine und kurze Kanäle $z u$ denselben. Mit den Geisselkammern wird das abfuhrende Kanalsystem eingeleitet; an ihm kann man ebenfalls kleinere radial verlaufende und grössere longitudinal verlaufende Kanäle unterscheiden. Die grösseren Kanäle münden in den Kloakalraum, und dieser durch die Oskula nach aussen. Die Geisselkammern liegen dicht neben einander; sie sind sack- oder birnformig gebaut (Fig. 5 u. 6); ihr Längsdurchmesser beträgt $0,045 \mathrm{~mm}$, ihr Querdurchmesser 0,03 $\mathrm{mm}$.

\section{Die zelligen Elemente.}

I) a s Ektoderm.

Das liktoderm der Spongien, zuerst von F. E. S chulz e ${ }^{1}$ ) an Sycandra raphamis $H$. nachgewiesen, besteht aus cincm

17) O. Schmidt, Amorphina genitrix is7o. S. 4 .

13) Vosmater, Porifera 1S86. S, 130.

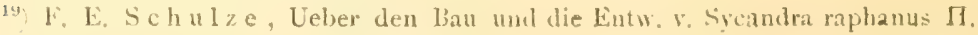
Zeitschr. f. wiss. Zoologie. Bd, XXV. I875. \&. 247. 
cinsehichtigen Pattencpithel. Die Zellen des Epithels, das sich sowohl aul cler Oberfliche des sanzen Schwammes findet, als auch dic zu- und abführenden Kanäle auskleidet, kömnen mehr oder lleniger regelmässig gebaut sein. Nicht bei allen von mir untersuchten lixemplaren von Hal. panirea gelang es mir, das liktoderm mit Sicherheit festzustellen. IVo ich es aber bemerken lionnte, (lige. 4) bestand es aus + bis 6 seitigen \%ellen, dic nur cinen seringen protoplasmatischen Inhalt enthiciten. Dic Zellen haben im Durchmesser cine Grösse von 0, OI I mm. Der Kern ist rundlich oval, liegt ziemlich in der Mitte der Kelle; scin Durchmesser beträgt $0,004 \mathrm{~mm}$. Der Nachweis des Plattenepithels geschah durch Anwendung der von F. L: S ch ul l e empfohlenen Silbermethode. Chromosmiumessigsäure oder r \% (Osmiumsäure vermögen jedoch auch die Zellgrenzen hervorzurufen. In cinem lialle erhielt ich das Fipithel sehr schön durch lixiren des Objects in einem Gemisch von Alkohol und Sublimat und Färben in Boraxkarmin.

Mesoderm.

Das Mesoclerm von Herl. proniced enthält verschiedenc zellige Bestanclteile. Auf cinem diinnen mit eincm beliebigen Farbstoff behandelten Schnitte bemerkt man unter dem Vikroskope eine Gewebschicht, die fast ganicht gefarbt ist und eine intensiv gefirbte Gewcbschicht. Dic crstere stellt dic hyaline Grundsubstanz dar, die zweite enthält das Geissclepithel, das ich beim Entoderm näher zu besprechen habe. In die hyaline Grundsubstanz sind dic Zellen des Mesoderms eingebettet. Bei Betrachtung eines Schnittes springen uns zuerst in die Augen langgestreckte spindelfömige Zellen. Sic nchmen leicht liarbstoff auf, wodurch sie sich von der ungefärbten Grundsubstanz deutlich abheben. Ihre Länge beträgt $0,05 \mathrm{~mm}$ ( Figr. $7 \mathrm{~b}$ ), die grösste Diclie $0,03 \mathrm{~mm}$. Lin Kern ist stets vorhanden; er sieht hell und bläschenformig aus, seine Form ist elliptisch, seine Grösse beträgt 0,0032 mm. Der Kén enthält ein dunkler gefäbtes Kemkörperchen von rundlicher Gestalt und I "Grösse. Dic /ellen haben ein feinkörniges Ausschen. Ist der Schwanm von einer Haut umgeben, so enthält sie diese spindelfömigen Zellen in srosser Zahl. Zahlreich funden sie sich atuch in der Umgebung der Kanäle und cler Kloake, vereinzelt 
zwischen den Geisselkammern. Strecken sich die /ellen bedentend in die Länge, sodass ihre Ausläufer eine beträchtliche Grösse erreichen, so haben wir es mit kontraktilen Faserzellen zu thun. Es ist natirlich schwierig, zwischen Spindelzellen und kontralitilen Faserzellen cine Grenze zu ziehen. Man trifft sic am häufigsten in der Oberhaut, wo sie senkrecht zur Peripherie verlaufen und in der Umgebung der Poren und Oskula, wo sie ringförmig angeordnet sind. Aus ihrer lage im Gewebe und ihrer Gestalt folgt, dass es Zellen sind, die den Muskelzellen höherer Tiere entsprechen. IF E. Schulz $\mathrm{e}^{20}$ ) hat sie kontraktile liaserzellen genannt, weil er die Bezcichnung ,Muskelzellen" nur auf solche Zellen angewandt wissen will, die mit Nervenzellen in Verbindung stehen.

Neuerdings wollen R. von Lendenfeld ${ }^{-1}$ ) und Solla s ${ }^{22}$ ) mit Sicherheit nervöse Elemente sowohl bei Kalk- als auch bei Kieselschwämmen nachgewiesen haben. v. Lendenfeld unterscheidet sensitive Zellen, Ganglienzellen und Nervenfasern. Die sensitiven Zellen sollen aus Ganglienzellen entstanden sein, letztere beschreibt er, wie folgt ${ }^{23}$ ): : the body of the cell is irregularly bulbous and extends in the shape of fine processes in every direction. It appears, that some of the processes connect these cells with the spindle shaped sensitive cells, they appear as nerve fibres. The cells themselves are situated very close together and their processes often touch. The nuclei of the cells are regularly splicrical. I consider them as ganglia cells. Aehnliche Zellen habe ich bei Hal. panicea gefunden; (Fig. 7a.) in der Nähe der ()berfläche des

2) F. E. Seln 1 z e, die Familie der Aplysinidae, Zeitschr. C. wiss, \%oulogie XXX, 187S, S. 394.

21) R. $\mathrm{r}$ Le n d e $\mathrm{fel} d$, The vestibule of Dendrilla carernosa. The Pruc. of the limn. Soc, of New South Wales. Vol. X. ISS5.

Synocil's, Simnesorgane d. Spongien. Zoolog. Anzeigr ISS7 S. $1+2-I+5$.

Beitrag zur Kenmtnis des Muskel- und Nervensystems der Hornspongien. Silzungsb. d. Kgl. Preuss. Akatd. d. Wissensch. 1SS5. 2. S. Ior5-1020.

Experimentelle Linters, iiber d. Physiol. d. Spongrien. Zeitsch f. w. Zoologie likl, +8. ISS9. S. 64 S.

$\left.{ }^{22}\right)$ W. J. Sollas, Tetractinellida. Reports on the Scientific Results of the voyage of II. M. S. Challenger. Zoolongy Bd XXV, p. XI.IS.

2:3) R. v. J,endenfeld, Die Chalineen des australiscluen Ciehiets. \%onl. Jahrbicher $1857 . \quad$ - 7.32 . 
Schwammes und in der Ungebung der Kanile. Sie sind von rundlicher melur oder weniger unregelmässiger Gestalt, ihre Grösse beträgt $0,01+\mathrm{mm}$. Ein Kern ist stets vorhanden, $\mathrm{cr}^{\circ}$ ist rundlich, $0,003 \mathrm{~mm}$ gross; im Kern ist cin Kernkörperchen von I "Grösse deutlich erkennbar. Die Zellen haben lange Ausläufer, die miteinander in Verbindumg stehen können. An einer Stelle in der Umgebung des Kanals fand ich 7 solcher \%ellen nebencinander liegend. Einen \%usammenhang der Ausläufer mit den kontraktilen Faserzellen komnte ich nicht mit Sicherheit feststellen. In wieweit man es hier mit Bindegewebszellen oder mit Anfänosen nervöser Eilemente zu thum hat, ist schwer zu entscheiden; ihrer sanzen Beschaffenheit nach machen dic Zellen den lindruck ron Ganglicnzellen, wie sie sich bei niederen Tieren finden.

Eine weitere Zellform des Mesoderms sind dic amöboiden Wanderzellen. Sie liegen in Mesodern zerstrent, sind von rund. licher oder ovaler Gestalt und erreichen eine Grösie von 0,01 2 mm.

Nacht man Schnitte durch jüngere Teile eines Schwammes, so sicht man Zige von parallel verlaufenden spindelförmig lansgestreckten \%ellen das Schwammgewebe durchset\%en. Dic Zïge können eine Länge von 0,2 mm und eine Breite von 0,05 mm erreichen. Die einzelnen \%ellen sind nicht dicht ancinander gelagert; kleine Lüclien zwischen ihnen sind erkennbar. Ihre Grösse beträgt $0,03 \mathrm{~mm}$, ihre Breite $0,005 \mathrm{~mm}$; ihrer Beschaffenleit nach sind sic grobliönig Jin Kern scheint sets vorhanden $z u$ scin; er hat ein bläschenfömiges Aussehen, sodass das dunkler sefärbte Kernkörperchen leicht erkemubar ist. 1)as Auftreten von jungen Nadeln in diesen /ellen lässt uns nicht im /Weifel, dass wir es hier mit Silikoblasten zu thun haben. I.eicler war esmir nicht moglich, Anfangstadien in cler Bildung von Nadeln $7 u$ beobachten. Nach neueren Untersuchungen von No/1\%.1) streckt sich dic Zelle, dic zur Bildung einer Nadel schreitet, zuerst in die Iänge, wobei ihr Inhalt sich aufhellt. Dann zieht ein dunkler cinfacher Strich von dem cinen Eunde der Zelle mitten rurch bis zum anderen, olne dass man Konturen ciner Nadel bemerkt. Das Auftreten dieses Strichs

24 F. C. No11, Beitrïge zur Naturgeschichte der Kieselschwämme I. 1)es-

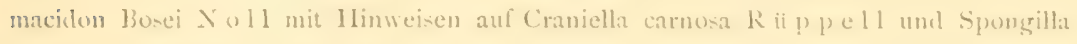

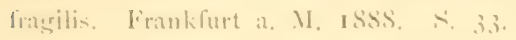


deutet er als Anlage des Centralfarlens, um den die Kieselsubstanz alogelagert wird. Die späteren Stadien habe ich selbst zu beobachten Gelegenheit gehabt. Je grösser die Nadel wird, desto mehr nehmen die spiculabildenden Zellen an Grösse ab.

Als letzte Zellform im Mesoderm will ich die Eizellen erwähnen. (Fig. 7 c.) Sie fallen clurch ihre Grösse und ihr begieriges Aufsaugen von Farbstoffen auf, Sie sind von runclicher, mehr oder weniger regelmässiger Gestalt, o,or $8 \mathrm{~mm}$ gross. Der Kern ist rund, $0,003 \mathrm{~mm}$ gross. Spermaballen habe ich nicht bemerkt. Obgleich ich zu verschiedenen Jahreszeiten frische Exemplare dredgte, so habe ich dennoch Entwicklungsstadien nicht beobachten können.

\section{Entoderm}

Das Entoderm wird aus den Geisselepithelzellen gebildet; sic sind cylindrisch gebaut; ihr Querdurchmesser beträgt $0,003 \mathrm{~mm}$, ihr Längsdurchmesser $0,006 \mathrm{~mm}$. Die Geisseln sind 0,03 mm lang.

Bisher nahm man an, dass die Geisselkammern Höhlungen im Schwamme seien, auf deren Oberflïche die Kragenzellen nebencinander stehen. $\quad \mathrm{v}$. Le n de $n \mathrm{fel} \mathrm{d}^{2}{ }^{25}$ ) kommt nach seinen neuesten Untersuchungen zu dem Resultate: dass dem nicht so ist, sondern dass vielmehr der Raum zwischen den Kragenzellen von einer durchsichtigen der gewöhnlichen Grundsubstanz der Zwischenschicht der Spongien sehr ähnlichen Substanz ausgefüllt sei. Mit anderen IVorten die Kragenzellen stehen nicht frei auf cler Oberfläche der Zwischenschicht, sondern sind in dieselbe eingesenkt«. Bei Hal. panieg habe ich Räume zwischen den einzelnen Kragenzellen nicht bemerken können; es ist daher schwer zu entscheiden, ob sie auf der Ziwischenschicht stehen, oder in dieselbe eingesenkt sind.

\section{Gems: Renierd. Schmidt.}

Bröcklige meist klumpige Massen. Kanalsystem rom dritten Typus. In der Regel bedeutende Subdermalhöhlen. Skelet aus vier- oder fünf- bisweilen drei- oder mehrseitigen Maschen gebildet.

25. v. I, e n d e $n \mathrm{fel}$ d, Notiz iiler den Ban der Geisselkammem der Sjongien. Zoologe. Anz, I?, 12, I $889 \%$. S. 36 I. 
Die Vadeln hiingen nur an den linden nittelst Sịnngin zusammen. Spikula indic. ac ${ }^{2}$ oder $\mathrm{tr}^{2}$. Nittelneer.

2. Species Remiera aquateductus. Schmidt.

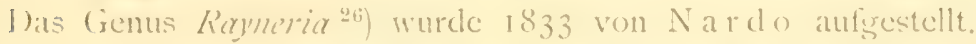

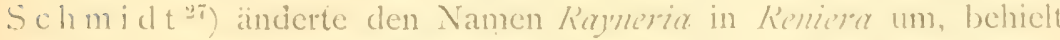
aber im allegemeinen die Merkmale, wie sie Nardo gegeben hatte. bei. Von Remerer aquateductus, I862 von ihm ais neue Species in das System cingeführt, gicbt er folgende Diagnose: "Re'llira fistulam vel simplicem vel ramosam imitans. Spicula ambabus extromitatibus acuminata in modum retis per totum corpus dispersa sunt. Color vel e cocrulen vei e rubro violaceus . Eine Oberhaut will er nicht bemerlit haben. Bei einigen lixemplaren habe ich aber cine solche sefunden; wir sehen auch hier, wie bei Ital. pernicier, wic wenig (iewicht auf das Fehlen oder Vorhandensein einer Oberhat \%u legen ist. 1. Al. Norman ${ }^{25}$ ) beschreibt in dem Supplementband zu Bowerbank s Monographic der Britischen Spongien einen Schwamm Malichondriar condenser. den ich mit Remierea aquedeductus fuir idenLisch halte.

(Fïg. S.) Rénierer aquequductus ist ein Schwamm, eler in verschiedenen frormen auftritt. Meistens ist er röhrig; in diesem laalle kann er cine ofler mehrere Röhren besitzen. Die Röhren erreichen cine Höhe $r o n 3 \mathrm{~cm}$, eine Dicke von $1 \mathrm{~cm}$, nach oben 7.4 findet cine Verjuingung statt. Die (Oskula, wenn vorhanden, haben einen Durchmesier ron $\mathrm{I}-2 \mathrm{~mm}$. In selteneren fällen hat kem. aquacduchus cine unregelmässige Gestalt, besonders dann, wenn sic auf Agen (I/hyllopherer) Krusten bildet. Dic Farbe variint sehr. Am häufigsten ist sie bläulich oder rötlich; an einem und demselben Exemplar zeist oft eine Rölure eine melur bläuliche, eine andere eine mehr rötliche lärbung. Weissliche Exemplare sind selten. Eine Oberhaut kann fehlen oder vorhanden sein; ist letzteres der Fall, so sitzt sic dem Innenparenchym nur lose auf und spannt sich

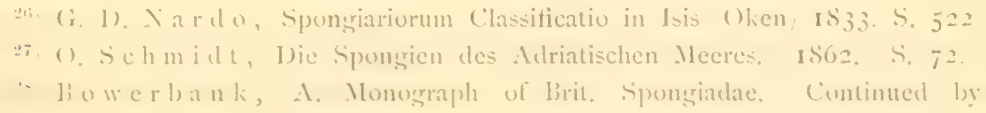

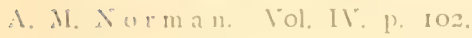


zwischen den hervorstehenden Nadeln zeltähnlich aus. Dic Nadeln bilclen sowohl in der Oberhaut, als auch im Innern des Selnwammes ein Netzwerk, dessen Maschen drei- oder vierseitig sind. Die Seiten der Maschen bestehen nicht aus mehreren nebencinandergelagerten Nadeln, wic bei Hal. panicea, sondern aus nur je einer Nadel. An den Spitzen sind die Nadeln durch Spongin verbunden. Diese Anordnunng der Nadeln und ihre Verbindung an den Spitzen durch Spongin, ist für das Genus Reniere charakteristisch. (Fig. 9) Dic Nadeln des Skelets sind an beiden Enden zugespitzt, gebogen oder in selteneren Fällen ein- auch zweimal seknickt unter einem Winkel von $150^{\circ}$. Nach Schulze und v. Lendenfeld 29$)$ werden sie mit Amphitorn bezeichnet; ihre Länge beträgt 0,12 bis 0,168 mm, die Dicke $0,006 \mathrm{~mm}$. Diese Nadeln bilden dic Grundnadeln. Daneben finden sich noch Amphistrongyle. Sie sind etwas kleiner als die vorigen. Drittens liommen noch Stylc vor.

$$
\text { Kanalsy stem. }
$$

1)as Kanalsystem stellt einen Ueberganes vom dritten zum vierten Typus dar, eine bestimmte Grenze lässt sich nicht zichen. Die zufuihrenden Kanäle, die bisweilen radiär verlaufen, entsenden \%u den Geisselkammern kleinere Kanäle. Die Geisselkammern sind kugelig; ihr Durchmesser beträgt $0,03 \mathrm{~mm}$; sic münden entweder dirckt oder vermittelst kleinerer Kanälchen in die abfuhrenden Kanälc.

liktoderm.

Das einschichtige Plattenepithel auf der Oberfläche und an den Kanälen weicht im Bau nicht besonders ab von den, wic ich es bei Ilal. panicea beschrieben habe.

$$
\text { Mesoderm. }
$$

Das Mesoderm enthält als charakteristische zellicre (icbilele die amöboiden Wanderzellen und spindelförmigen Zellen. I at-

2a) F. E. Sehulze und $v$, Lendenfeld, Leber die liezeichnung der spongiennadeln. Berlin 1889 
zwischen breitet sich dic (roundsubstan\% alus, die nicht se hyalin ist, wie bei llat. panieer. sondern kömchenreicher. Dic amöboiden Wanderzellen sind an Grösse und Gestalt verschieden. Die spindelfömigen Zellen finden sich besonders zahlreich in cler Oberhatut. Im Innenparenchym licgen sic zerstreut zwischen den Geisselkammern, regelmässiger angeordnet in der Umgebung der Kinaile. Von anderen Zellen des Mesoderms sind die Silicoblasten zu erwähnen, die besonders in jüngeren Schwammteilen in Küugen von $0.7 \mathrm{~mm}$ I änge und $0,06 \mathrm{~mm}$ Breite das Gewebe durchsetzen. (Fig. 10.) Lizcilen und Spermaballen habe ich gleichfalls beobachten können. Die Eizellen tragen das charakteristische Geprägege einer Schwammeizelle. Sperma findet sich in kugeligen Ballen, dic etwas kleiner als dic Geisselkammern und im äbrigen nicht leicht von ihnen $z u$ unterscheiden sind. F. E. S c h ul z $e^{30}$ ) hat ähnliche Gebilde bei Plakina monoloplua beschrieben.

\section{lintoderm.}

Dasselbe besteht aus rundlichen Geisiclepithelzellen, die cine Grossc von 0,004 mm haben.

3. Species: Reniera (Isodyctia) itratans. bo w e r b a $n \mathrm{k}$.

Diese Spongie wurde allem Anscheine nach zuerst von Bow r a a $\mathrm{k}^{31}$ ) als Isodyctia rarians beschrieben. Das Genus Isodyctia wurde I 864 von ihm aufgestellt ${ }^{3 *}$ ). Iis soll Spongion umfassen, deren Skelet keine Sponginfasern enthailt, sondern aus cinem symmetrischen Netzwerk von Nadeln besteht (Slieleton without fibre, composed of a symmetrical network of spicuia). lis würde also mit dem Genus Remierd Schmiat identisch scin. Womn man jedoch den Angaben Schmidt's ${ }^{33}$ ) und Vosmaer's ${ }^{31}$ ) folgt. so enthält das Genus Isodyctir auch Spongion mit deutlich ausgeprägten I Iornfasern. Vosm a c r erkennt deshalb das Genus sciner

3.) F: E. Schulze, lamile der l'kakiniden. Zeitschr. I. Wissensch Zoolog. biel 3t. 5. $+1+$.

क. I

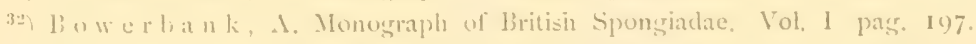

:3 (). Schmidt, Grundzitge einer Spongienfauna des atlant. (iebiets.

$1 \mathrm{~S} ; 0, \therefore 1 \%$.

st fusmacer, l'orilera isso. \&. 210. 
heterogenen Gebilde wegen nicht an. Unter den $S$ ch m i d t'schen

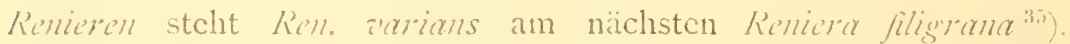

Ren. ararans ist ein Schwamm, der in der Kieler Bucht eine Höhe von $12 \mathrm{~cm}$ erreicht. (IFig. I I.) Er bildet eine weitverzwcigte Staude mit fingerförmigen Fortsätzen. Die einzelnen Fortsätze sind dïnn und schlaff, von nur geringem Zusammenhalt; aus dem Wasser gcnommen, können sie sich nicht aufrecht erhalten; die Farbe ist weisslich grau, bisweilen rötlich. Eine Oberhaut ist stets vorhanden, doch sitzt sic dem Innenparenchym nur lose auf. Osliula sind sclten und dann unregelmässig zerstreut. Das Skelet besteht hauptsächlich aus Amphitornen, die $0, \mathrm{I} \mathrm{mm}$ lang und $0,007 \mathrm{~mm}$ dick sind. (Fig. I2.) Bisweilen erreichen sie auch eine Länge von $0.33 \mathrm{~mm}$; ihre Dicke ist dieselbe, wie bei den kleinercn Nadeln. Häufiger sind Style anzutreffen von $0,09 \mathrm{~mm}$ Länge. Die Nadeln bilden in der Oberhaut ein symmetrisches Netzwerk, dessen einzelne Maschen vierseitig sind, ähnlich wie bei Ken. aquacductus. An den Spitzen sind sie durch Spongin verbunden. Anders ist die Anordnung der Nadeln im Inneren des Schwammes. Hicr bilden die Nadcln, ebenfalls durch Spongin verkittet, kein so schön ausgeprägtes Netzwerk, wie an der Oberfläche, sondern Reihen, die von unten nach oben d'n Schwamm durchziehen. Dadurch, dass das Spongin die ganze Nadel ihrer Länge nach ïberzieht, crscheinen die Nadeln wie in eine Sponginfaser eingebettet. In diesen Sponginfasern können auch mehrere Nadeln nebeneinander liegen. Rén. zarians vereinigt in sich Charaktere der Gattung Reniera insofern, als die Nadeln an cler Obcifäche cin ausgeprägtes Netzwerk bilden, andererseits aber auch Merlimale der Gattung Chalim, da in ailteren Teilen des Schuammes die Nadeln in mehreren Reihen nebeneinanderliegend ganz von Sponginsubstanz umhüllt sind. Ken. z'rrians bildet also cinen Uebergang von den echten Renieren zu den eigentlichen Chaline'n, und man könnte sie auch dem Schmidt'schen Genus Chalimula zurechnen.

3i) $0.5 \mathrm{eh} \mathrm{midt,} \mathrm{die} \mathrm{Spongien} \mathrm{des} \mathrm{adriatischen} \mathrm{Meeres.} \mathrm{IS62.} \mathrm{S.} \mathrm{7t.}$ 
Lanalsystem.

Dats Lanalsystem gehört dem dritten Typus an. Dic (seisselliammern sind mregelmässigy angeordnet; bald liegen sic in Matufen nebencinander, bald sparlich \%erstrent. Ihre Gestalt ist liusclig; ilure Grössc butriggt $0,02 \mathrm{~mm}$.

\section{lik to der 11 .}

bie \%ellen des liktoderms zeigen in ihrem bau nichts chanaliteristisclues.

\section{Hesoderm.}

1)ic hyaline Grundsubstan\% ist reich cntwickelt. Nur spärlich finden sich in ihr amöboide Wanderzellen und spindellörmige Zetlen. Auf dicsen Umstand ist wohl auch dic grosse Schlaffheit des Schwammes, besonders die der fingerförmigen Fortsätzc zurickzufihren. An den Spitzen der Nadeln, oft noch im Zusammenhang: mit dem Spongin, habe ich /ellen bemerkt, dic ich tür Spongo blasten halte. Sic sind feinkornig, enthalten einen hellen Kern, der ein dunkles Keruliörperchen umschliesst. An Gestalt ähneln

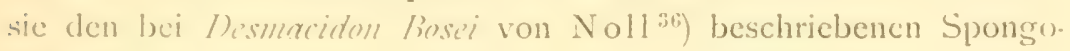
blasten.

\section{$1: 11+1) d<111$.}

bie \%ellen des Entoderms sind denen von Rem. aquacductus ihlulich,

\section{Die (Malineen.}

Der Name (\%oline (urspringlich Helina) wurde von (i) it in t cingeführt und zwar an Stelle von Fleming's Falichondrix. bowerbank ${ }^{37}$ ) stellte I 864 Chalina als Unterordunng der Ordnung Keratosi mit folgenden Merkmalen auf: \$Skeleton fibrous Jibres keratose, solid, cylindrical and interspiculate. Rete symmetrical; primary lines radiating from the basal or axial parts of the sponge to the distal portions. Secondary lines of fibre at about

.46 Nol1, beiträge zur Naturesesch. 1. Kiesclschwämme. I. 1)esmacidon bosei. lirankf. a. $11,18 s 8$. is +2.

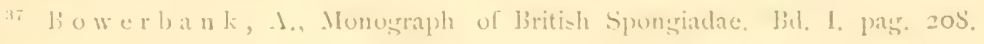


right angles to the primary ones. $\Lambda$ ss Typus fiihrt er Chaline oculate an, die aber nach $\mathrm{Schmidt}^{38}$ ) nur in den ailtcren 'Teilen ein wirkliches Hornfasernetz, in den jingeren Teilen ein renierenähnliches Netzwerk besitzt.

In den Spongien der Küste von Algier stellt Schmidt cine cigene lamilie der Chalineen auf, da das Genus Chalina Bow erbank \% heterogene Bestandteile aufweist. Lir teilt dic Chalinern cin in dic Gaitungen I. Siphonochalina: "verzweigte oder unverzweigte Röhren; die Oberfläche dicht, indem zwischen den wenig hervorragenden Enden der radiiren Fasern, ein feineres dichteres Fasernetz sich ausbreitet, 2. Chalinula: »äusserer Habitus und lockerer Zusammenhang der echten Renieren, aber cin Hornnet\%, welches einen grossen Teil der Fasern vollständig umbüllt, 3. Sclerochalina: Habitus von Siphonochalina, aber mit gröbcrem, unregelmässigerem Netze." 4. Pachychalina: "Fasern aus vielen Reihen von Nadeln gebildet, welche durch Hornsubstanz fest verbunden sind." Diese Einteilung hat er in den Grundzïgen ciner Spongienfauna des atlantischen Gebiets I870 mit cinigen Abänderungen beibchalten. v. Lendenfeld ${ }^{39}$ ), der die Chalineen des australischen Gebietes und Keller ${ }^{40}$ ), der die (Halineen des roten Neeres beschrieb, haben sie um viele neuc Species vermehrt. Vosmaer behailt auch im Al!gemeinen die Einteilung der Chaline'n, wie sie Schmidt gegeben, bei. Das Genus Chalimula ist nach ihm von Chalina zu wenig verschieden, als dass es abgetrennt werden kann. Wenn man aber 7.4 der Gattung Chalinule alle die Species rechnet, dic nicht in allen Teilen ein ausgeprägtes Fasernet\% besitzen - Uebergangsformen von Renieren zu den Chalineen - und zur Gattung Chalina diejenigen, die cin solches besitzen, so ist die Trennung der beiden Gattungen wohl durchfuhrbar. Nach dem Bau des Hornfasernetzes zerfallen die Chalineen in Chalinidae reticulatac mit netzförmigen Fasern und (halinidae den-

3s) O. Sch midt, die Spongien d. Kiiste von Algier. 2. Supplement zu den Spongien d, adriat. Meeres. I 866. S. 9.

$\left.{ }^{39}\right)$ v. L e $\mathrm{n}$ d e $\mathrm{n}$ feld, die Chalineen d, australischen Gebiets. Zoolog. Jahrb. IS87. S. $739-743$

4) Ke 11 e r, d. Spongienfiuna d. roten Meeres. Keitsclur. f. wiss. Zoologric. I3d. $48,1889$. S. $374-394$. 


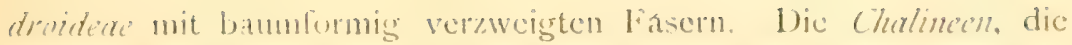
in der ()stsee vorkommen, sind Chalimula ornlmm Schmidt, (Vur-

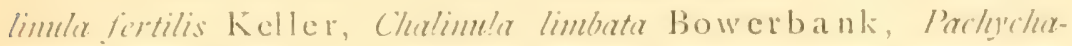
lima rustica Schmidt; sie gehoren zu den Chalinidar reticulatas.

Gimes Chalimila. Schmidt.

Acusserer Ilabitus und lockerer Zusammenhalt der echten k'mi'r"l, aber ein Hornuctz, welches einen grossen leil der liasern vollstindig umhillt.

(Iig. 13.) f. Spere: Chal. orulum. Schmidt.

I)ic Gattung (Kalimula wurde von Schmidt I $868^{\text {") }}$ ) aubgestellt. Sic umfasst Chaline's von renicrenähnlichem Bau. Dic Jadeln liegen in Sponginfasern eingebettet, die nur in älteren Teilen des Schwammes stärlier ausgebildet sind. Die Gattung Chalimula bildet also den Uebergang von Reniera zu Chalina. Bestimmte Grenzen lassen sich nicht ziehen. „Der Charakter der (Yuline'\%, sagt Schmidt. wird in diesen Uebergängen so verwischt, dass man nur noch durch cinzelne faserartige und Nadelreilicn enthaltemle Sarkodestringe in dem sonst ganz Reniera-artigen Schwamme daran crinnert wird. « Die I 870 von Schmidt ${ }^{4 \%}$ ) aufgestellte Species Char. omulum von der grönländischen Küste charakterisirt er folgendermassen: "Sic siedelt sich in Gestalt cincs kleinen lockeren, laubförmigen oder ellipsoidischen Körpers auf Algen an, hat eine poröse Oberfiche und $1 / 2-3 / 4 \mathrm{~mm}$ weites Oshulum. Dic Nadeln stumpf-spitz, o, I $6 \mathrm{~mm}$ lang, liegen im Innern dicht und unregelmässig. Dazwischen aber ziehen sich radiäre Züge zusanmengekitteter Nadeln zur Oberfliche, wo sie in Pinsclchen etwas vorragen. Dieser Diagnose habe ich nur weniges hinzuzufugen. Dic radiären Züge sind $0,03 \mathrm{~mm}$ dick; nach der Oberfäche zu nehmen sic an Dicke ab. Viihrend im Inneren des Schwammes $4-5$ Viadeln neben einander durch Spongin verkittet liegen, werden sic

") (1) Selt midt, d. Spongien d. Kiiste von Algier. S. Supplementb, zu den Spungien d. adriatisch Mecres. I86s. S. 7

1:. (). Sichmidt, Grundzighe einer Spungientauna des atlantischen (iebiets. 1870. ‥ 38 
der J'ripherie zu späirlicher. Die Spitze der Sponginfaser, die O,I4 bis 0,2I mm über die Oberfläche hinausragt, enthält nur noch cine, hichstens zwei Nadeln. Senkrecht zu diescin radiären Sponginfatern verlaufen concentrische, die diunner sind und Nadeln nur in geringer Anzahl enthalten. Neben den stumpfspitzen Nadeln kommen noch in den Sponginfasem an beiden Seiten abgerundete vor, Amphistrongyle, die Schmidt übersehen hat; sie sind $0,1 \mathrm{~mm}$ lang und finden sich nur vereinzelt. (Fig. I4.) Beide Nadelformen sind cntweder gerade oder leicht gewölbt. Die Dicke beträgt O,OI mm. Da mir nur Spiritusmaterial zur Verfugung stand, und dicses auch nur in geringem Masse, so habe ich mich auf das Studium histologischer Details nicht einlassen können. Das Kanalsystem gehört dem dritten Typus an.

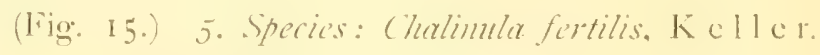
(Pluploliphonia fortilis, L, endentcld.)

In ciner Abhandlung Studien über Organisation und Entwicklung der Chalineen beschreibt Keller ${ }^{13}$ ) eine neuc Species Chalimula fertilis, die er bei Porto militare im Golf von Neapel gefunden hat. Als Hauptliennzeichen der neuen Species fülurt er folgende an: Der Schwamm ist vorzugsweise monozoisch und bildet $2-3 \mathrm{~cm}$ lange aufrechte Röhren oder Kegel mit einem Oskulum an der Spitze, dessen Durchmesser 2-3 mm betragt, der Schwamm zeichnet sich im Leben durch grosse Zartheit aus, und frisch aus dem Wasser genommen, liönnen sich die einzelnen Röhren liaum aufrecht erhalten; nach dem Abfliessen des im Schwamme reichlich enthaltenen Wassers wird derselbe schr schlaff und zerreissbar. In getrocknetem Zustande ist derselbe elastisch. Neben monozoischen Formen liommen auch grössere oder likinere Polster vor, deren Durchmesser selten über vier $\mathrm{cm}$ hinaus geht, und auf denselben crheben sich 2-4 fingerförmige Personen mit weitem Oskulum. Das Fasernetz besteht aus regelmässig um cinen Centralmagenraum angeordneten Radiärfasern, welche durch ein System von Kreisfasern verbunden sind. In diese Hornfasern eingebettet sind gerade

1:5 K $11 \mathrm{er}$, Studien iber Organisation und Entwicklung. der Chalineen. Zcitschr. 1. wissensch. Zuolngie. 13d, 33 ISSS. \$. $317-349$ 
oder schwach gebogene einfache Kiesclnadeln, welche bald an beiden Enden zugespit\%t, bald an einem oder auch an beiden linden abgerundet sind. Ihre Länge beträgt $0,092-0$, I mm. I)ie I)ermalflïche ist glatt. In seinem Werke »Die (.halinee"l des australischen Gebietes stellt $v$. Lendenfeld ${ }^{44}$ ) eine neue Gattung I'hylo/iphomiur auf und sagt: von schon bekannten Arten gehört hierher (halimulir fertilis K $\mathrm{K} / \mathrm{l}$ e r. Da jedoch der Speciesname Chalimuler firtilis älter ist, so muss er beibehalten werden.

$$
\text { Kan a lsystem. }
$$

Das Kanalsystem zeigt Uebergänge vom dritten zum vierten 'Iypus. Die zufihrenden Kanäle entspringen nicht direlit den Hautporen sondern sogenannten Subdermalhöhlen, die sich zwischen der den Schwamm überzichenden Haut und den Innenparenchym ausgebildet haben. Ke $11 \mathrm{cr}$ unterscheidet am Kanalsystem von Cluxl. fertilis einen centralen und einen peripherischen Abschnitt. Der crstere entspricht dem abführenden, der letztere dem zufuhrenden System. Dic Geisselkammern sind kugelformig; ihr Durchmesser beträgt $0,02 \mathrm{~mm}$.

Das Elitoderm besteht aus einem einschichtigen l'lattenepithel, dessen '/ellen in Grösse und frorm variiren; der Kern ist rundlich.

Die Grundsubstanz des Mesoderms ist von homogener Beschaffenheit. In sie eingelagert sind spindelförmige \%ellen, die in der Ungebung der Poren sphinkterartig angeordnet sind, und amïboide IVanderzellen.

Dic fiormelemente des Entoderms sind die Geissel\%ellen. dic denen bei Reniere beschriebenen ähnlich sind.

\section{Genus: Chalina. Bow e r b a $13 \mathrm{k}$.}

Rölnigr, cylindrisch oder klumpig, Sponginfasern deutlich ansegebildet, ein Netzwerk bildend von oft ziemlich regelmässigquadratischen Maschen. Lakune gross. Spic. indic. ac ${ }^{2}$ (gelegentlich auch tr. ac.). Atlantischer Ocean, Mittelmeer, Arktischer Ocean.

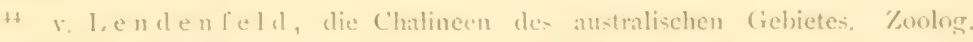
fatirt. rsiz. S. $7+4$. 
(Fig. I6.) 6. Spectes: Chalina limbata. Bow crbank.

(Spongia limbata. Joh ns to n).

Nach Lieberkïh ${ }^{45}$ ) finden sich die ersten Angaben iiber diesen Schwamm bei Johnston. Er selbst giebt dann eine kleine Beschreibung von Spongia limbata, die er bei Wismar in der Ostsee sefunden hat. Bowerban $\mathrm{k}^{46}$ ) reihte Johnston's Spongid limhata in sein Genus Chalina ein.

Sowohl Lieberkii hn, als auch $\mathrm{B}$ ow e r ba $\mathrm{nk}$ beschreiben Chalina limbata als cinen Schwamm, der selten die Grösse einę Haselnuss iiberschreitet und Fucus resiculosus aufgewachsen ist. In so kleinen lixemplaren habe ich ihn in der Kieler Bucht ebenfalls gefunden; doch glaube ich, dass wir es hier mit jungen Schwämmen zu thun haben, da ich diese kleinen Exemplare nur im Dezember und Januar antraf. Im Sommer und Herbst waren die von mir sedredgten Schwämme bedeutend grösser. Während dic kleinen oft nur cine flache Scheibe auf Fucus bildeten, oder nur e i n e Röhre darstellten, waren die grösseren stets vielröhrig, oder zeigten, wenn sie einröhrig waren, doch schon die Anlage einer zweiten Röhre. Die Röhren, die eine Höhe bis $4 \mathrm{~cm}$ erreichen kömmen, erheben sich auf einer polsterartigen Unterlage. Sic liegen entweder in ciner Ebene oder wachsen nach verschicdenen Richtunsen hin. Im extremsten Falle findet alsdann eine strahlige Anordnung der Röhren statt, die miteinander verwachsen können, sodass der Schwamm ein klumpiges, massiges Aussehen erhält, das Aussehen ciner Kugel etwa, auf deren Oberfläche sich die Oskula befinden. Der Durchmesser eincr einzelnen Röhre beträgt $10-12$ " $\mathrm{mm}$, " nach oben findet eine Verjüngung statt. Die Oskula sind $2-3 \mathrm{~mm}$ gross. Die Farbe ist braun oder schmutzig gelb. Durch das Hervorragen der Hornfasern uber die Oberfläche hinaus fühlt sich der Schwanm rath an. Zwischen den Hornfasern spannt sich eine Haut aus, in der sich rundliche oder ovalc Poren befinden, dic $0,02-0,05 \mathrm{~mm}$ gross sind.

An einem ausgeprägten Hornfasergerist der Chalinidae roti-

45) Lieberk ii hn, Beitrigre zur Anatomie der Spongien Archir f. Anat. und Physiologie 1857. S. 398.

4) Ho if e r bank, A Monograph of liritish Spongiadae. V. I, II. pagr. 373. 
culatar unterscheidet man radiäre, konzentrische und longitudinale lasern (nach Bow e rbank primary secundary aud longitudinal lines). Die radiären Fasern bezeichnet man auch als Hauptfasem oder Fasern erster Ordnung, die konzentrischen und longitudinalen als Verbindungsfasern oder Fasem zweiter Ordnung. Dic radiären Fasern gehen vom Mittelpunkte des Schwammes zur Peripherie; die konzentrischen schneiden die radiären unter einem bestimmten Winkel und verlaufen in horizontaler Ebene, die longitudinalen stehen senkrecht auf beiden. Bowerbank giebt den Winkel, unter dem sich die radiären und longitudinalen lasern bei Cha!. limbato schmeiden als einen Rechten an, doch ist dies durchaus nicht immer der Fall, besonders im Innern des Schammes trifft dies nicht zu. Hier ist das Hornfasernetz sehr dicht und unregelmässig; der Peripherie zu werden die Maschen weiter und bilden ziemlich reguläre Rechteclic. Dic Weite der Maschen an der Oberfläche beträgt 0,3-0,5 $\mathrm{mm}$, dic Dicke der Hauptfasern $0,07 \mathrm{~mm}$, dic der Verbindungsfasern $0,035 \mathrm{~mm}$. (lig. 57. ) In dic Hornfatsern sind die Nadeln eingebettet, die hauptsächlich Amphioxe, 0,098 mm lang, und 0,003-0,005 $\mathrm{mm}$ dick sind. (Fig. I 8.) Sic durchsetzen in Zügen, 15-20 nebeneinander liegend, die Hauptfasern. In den Verbindungsfasern sind die Nadeln spärlicher, gan\% fehlen sie aber nie. Die Nadeln in den Fasern bezeichnet man als Skeletnadeln im Gegensatz zu den zerstreut im Schwammgewebe liegenden Nadeln den sog. Fleischnadehn. Bei Chal. limbater unterscheiden sich die Fleischnadeln nicht von den Steletnadeln; sie haben im allgemeinen dieselbe Grösse und Gestalt. In vercinzelten lä̈llen habe ich Fleischnadeln von $0,3 \mathrm{~mm}$ Länge gefunden, ganz vom Aussehen der Nadeln von Halichondria panicea es ist nicht unmögrlich, dass sic als Fremdliörper von dem Schwamme aufsenommen sind. Wohl aber kann man bei Chal. limbata zwischen Dermalia und Parenchymalia unterscheiden. Die Dermalia sind ()xydiactine, Bowerbank hat sie übersehen. Er beschreibt sic bei scincr Isodyctia momaler unter dem Namen inflato-fusiformi-acerate. Die Oxydiactine sind etwas kileiner und schanker als dic Amphioxe.

1)as Kanalsystem gehört dem dritten Typus an. Dic Geisselkammern sind kusclig eiformig $0,035 \mathrm{~mm}$ gross. D)as Ektoderm 
besteht aus dem Plattenepithel, das nach dem gewöhnlichen Typus gebaut ist.

Von den Zellen des Mesoderms sind zu erwähnen dic spindelförmigen Zellen, die sich besonders zahlieich in der Nähe der Oberfläche des Schwammes und der Kanäle betinden, und die amöboiden Wanderzellen.

Eine besondere Aufmersamkeit luabe ich der Bilchung der Sponginfasern zugewandt. Nach If. Ii. Schulze ${ }^{47}$ ) entstehen bei den echten Hornschwämmen die Hornfasern durch die Thätigkeit der Spongoblasten »cylindrische oder birnförmig gestaltete membranlose kernhaltige Zellen, welche mit ihrem, der Faseraxe zugewandten quer abgestutzten proximalen Ende die Faseroberfläche direkt berihren, mit dem anderen abgerundeten Ende mehr oder minderweit in die umgebende Bindegewebsgrundsubstanz hineinragen". Es bildet sich zuerst ein Axenstrang, in dem sich gewöhnliche Bindegewebszellen zu strang- oder netzförmigen Zïgen anordncn, indem dic hyaline Grundsubstanz resorbirt wird. Auf dicse Iirstlingsproduktion der jungen gleichsam noch unreifen Spongoblasten folgt nun nach ihrer vollständigen Ausbildung zu cylindrischen radiär gerichteten Zellen - reifen Spongoblasten von ihrer proximalen Endfäche aus die schubwcise Ablagerung der Sponginlagen auf jenen zuerst gebildeten Axenstrang liei Chal. limbata habe ich analoge Erscheinungen beobachtet. lis bilden sich ebenfalls Zellen des Bindegewebes zu Spongoblasten um, indem sie in Strängen, - ähnlich wie bei Halichondria panirea und Reniera aquaeductus die Silikoblasten - die Grundsubstanz durchsetzen und die Sponginfasern ausscheiden. Die Spongoblasten von Chal. limbata sind aber anders gestaltet als die Spongoblasten der echten Hornschwämme, wie sie F. E. Schulze bei den Spongidae beschreibt. Sie sind von spindelförmiger Gestalt $0,025 \mathrm{~mm}$ lang mit einem rundlichen Kern; sic ähneln den Spongoblasten der Kieselschwämmc, wie Nol1 4s) bei Desmacidon Bosei beschreibt.

Die Zellen des Entoderms sind rundlich, O,O I mm gross.

$\left.{ }^{47}\right)$ F. E. S $\mathrm{c}$ h u $\mathrm{l} z \mathrm{e}$, die Familie der Spongidae. Zeitschr. f. wiss. Zoologrie. liil. X.1.11. Is;). $\rightarrow 0.35$.

is) Nol1, lieiträge zur Naturgesch. der Kieselschwimme. I. Desmacidon Busei Noll, Frablifurt a, M ISSS, S. 4.3. 


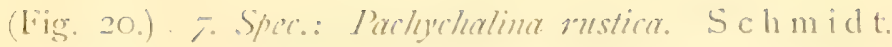

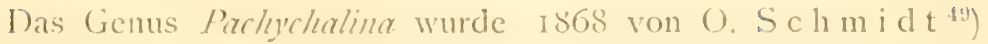
mit folgender 1)iagnose aufgestellt: : Fasern aus viclen Reihen von Nadeln gibildet, welche durch Hornsubstanz fest verbunden sind. Dic Specics l'. rustica besclueibt $\mathrm{cr}$ folgendermassen: " $3 \mathrm{~cm}$ hoch. papillenfömicy mit etwas verdicktem oberen knde und ganz unregelmäissiger, feinkrausiger Oberfläche, oder länglich kegelförmig struppig. Dic spitz-spitzen Nadeln sind 0, $16 \mathrm{~mm}-0,17 \mathrm{~mm}$ lang. In der Kicler Bucht erreicht $I$. rustica cine Jöhe bis $4 \mathrm{~cm}$. Mit scincm unteren, verschmailerten linde ist der Schwamm auf Fucns aufgewachsen. lir ist monozoisch von lederartiger Consistenz. Die radiaren Fasern sind $0,03 \mathrm{~mm}$ dick; sic ragen etwas über die Oberfäiche des Schwammes hervor, endigen aber nicht spitz, sondern in eine Kappe. Die Kreisfasern sind etwas dimner. Die in die Fasern eingebetteten Nadeln sind Amphioxe, 0,08 mm lang, (). Schmidt hat sie bei den Exemplaren an der Küste von Algier 0,16-0,175 mm lang gefunden. P' mstica zeigt in Bezug anf Nadellänge cin ähnliches Verhalten wie Hal. panicea, wo bei Nordsceexemplaren und bei solchen aus dem atlantischen Ocean dic Nadeln auch beträchtlich länger sind, als bei Exemplaren der Ostsee. Häufig habe ich neben den Amphioxen noch Oxydiactine gefunden, wie sie auch bei Chal. limbate vorkommen. (Fig. 21.) Das Kanalsystem gehört dem dritten Typus an. Dic Geisselkammern haben eine rundlich-eiformige Gestalt und sind 0,03 mm gross. In Bezug auf den histologischen Bau ist zu bemerlien, dass sich P. rustica nicht wesentlich von Chal. limbata unterscheidet.

\section{Familia Desmacidonidac.}

Skclet besitzt ausser den-stabfömigen Skeletnadeln noch zahlreiche sog. Fleischnadeln, wie Anker, Schaufel, Bogen etc, Die Nadeln entweder gan\% durch Spongin umgeben, oder ganz frei; nie teilweise hervorragend. Spongin oft stark entwickelt, Kanalsystem soweit bekannt vom dritten Typus.

4:) O. Sch midt, die Spongien tler Kiste rom Mlerier. 3. Supplem. zu den spomsien a, arratischen Meeres. Isos. s. S. 
Gemus: Esperella (Esperia). Es per.

Stabnadeln glatt oder gedornt. Schaufeln mit ungleichen Enden. Dazu können Haken und Bogen kommen. Sponģin meist deutlich. Mittelmeer, Arlitischer Ocean.

(Fig. 22.) 8. Spec.: Esperella (Esperia) lammgo. Sch mi d t.

Das Genus Esperia (von V os m a e ${ }^{50}$ ) in Esperella umgeändert, weil Esperia schon für eine Lepidopteron angewandt war) wurde von $\mathrm{Nardo}{ }^{51}$ ) in seiner Klassifikation der Schwämme aufgestellt. Die Species Esperella (Esperia) lanugo wurde I875 von $\mathrm{Sch} \mathrm{midt}$ in das System eingefuihrt; gefunden wurde sie auf der Expedition zur physikalisch-chemischen und biologischen Untersuchung der Nordsee im Sommer I872 im grossen Belt. Schmidt ${ }^{52}$ ) giebt folgende Beschreibung von ihr: „Rundliche Körper von weicher, flockiger Beschaffenheit mit cinzelnen Oskulis. Nadeln stumpf-spitz. Nadelziige nicht stark, zwischen ihnen viele isolirte Nadeln. Die Haken (Schaufeln) zeichnen sich aus durch ungewöhnliche Entwicklung des sonst beim Esperienhaken zuriickbleibenden Endes. Einzelne Haken sind nach beiden Enden völlig glcich entwickelt, wie bei Desnacidon arcifermm. Die Gestalt ist nicht immer rundlich, sondern bisweilen länglich. Die Farbe des Alkohol. praeparats ist bräunlich. Die Nadeln, die zu $0,04 \mathrm{~mm}$ dicken Zügen vereinigt sind, sind stumpfspitz, 0,224 $\mathrm{mm}$ lang und $0,008 \mathrm{~mm}$ dick; sie sind mehr oder weniger gebogen oder geknickt. Die Biegungsstelle liegt nicht in der Mitte, sondern mehr dem stumpfen Ende der Nadel zu. Daneben finden sich noch, frei im Parenchym liegend, sehr dunne, ebenfalls stumpfspitze Nadeln und spitzspitze Nadeln, o,4 $\mathrm{mm}$ lang und $0,0 \mathrm{~mm}$ dick, wie sie bci Halichondria panicea vorkommen. Charakteristisch für die Esperien sind dic sog. Schaufeln, 0,024 mm lang. (Fig. 23.) Wührend bei den übrigen Esperein die Platten an den Enden der Schaufeln immer ungleich gross sind, finden sich bei Est. lamugo auch Schaufeln, deren Enden gleich grosse Platten haben.

so, Vosmacr, Porifera 1887 . S. 454.

i1) Narko, Spongiariorum Classificatio in Isis (Oken) 1833. pas. 522.

5. Jahresbericht der Kommission zur wissenschaftlichen Lntersuchung der deutschen Necre fux die Jahre I\$72 u. $1 \$ 73$, berlin 1875 . 5 I 18. 
Neben dem aus Nadelziigen gebildelen Skelet besitzt lisp. lanuge noch cin aus Fasern gebildetes Geriist, das S Schmidt nicht crwihnt. \uf Querschnitten bemerkt man ziemlich in der Nitte des Schwammes eine kreisförmig verlaufende laser. (Fig. 24.) In dieser kreisformigen Faser, deren Durchmesser $0,35 \mathrm{~mm}$ beträgt, verlaufon $4-5$ radiär angeordnete Fasern, dic im Mittelpunkte verschmelzen, sodass cin (ieriist entsteht. Dic Dicke der Fisern, dic atus Lamellen zusammengesetzt sind, beträgt $0,03 \mathrm{~mm}$. In den durch die Fasern gebildeten Maschen sind \%ellenreste sichtbar; die cinzelnen \%ellen sind rundlich $0,005-0,007 \mathrm{~mm}$ gross. (Fig. 25.) Solche \%cllenkomplexe habe ich nicht nur in den Maschen des Geriistes gefunden, sondern auch frei im Parenchym liegend; sie waren von rundlicher Gestalt, und bei einzelnen kounte ich auf der Peripherie eine offenbar von den Zellen ausgeschiedene, junge, krcisrunde Faser bemerlien, die beständig auf Kosten der einzelnen Zellen wächst - diese Zellen scheinen also cine den Spongoblasten der llornschwamme analoge Thätigkeit zu entfalten. (Fig. 26.) Entstehen im l'arenchym nahe beicinander mehrere solche Ringfascm, dic sich mehr und mehr vergrössern, so müssen sie schliesslich aufeinander stossen; die sich berührenden Fasern verschmelzen miteinander und bilden so das obenerwähnte Geriist. Ein ähnliches Geruist beschreibt v. Marten s ${ }^{53}$ ) bei Spongia Comtarenii, M a rtens (Esperia Contarenii) $\mathrm{Schmidt}$ ): \# lis ist ein grobes Netz viclfach anastomosirender, derber, harter, milchweisscr libern, bis $2.11 / 3$ Linic im Durchmesser.

Dats Kanalsystem gehört dem dritten Typus an.

1)a mir nur Spirituspräparate zur Verfüung standen, so konnte ich auf die histologischen linzelheiten nicht näher eingehen.

Ordo: Spiculispongiac.

Skelet sehr selten fehlend. Skeletelemente, wenn vorhanden, vorwiegend selbständige Spicula, dic, wenn sic fest verbundene Slielete bilden, durch eigentimliche knorrige Auswiehse ineinander greifen, aber niemals in der IVeise durch Kieselplatten verschmolzen 
sind, wie bei den Hyalospongiae. Oft mittelst organischer Substanz in biindel zusammengelittet.

$$
\text { Subordo: () ligosilicina. }
$$

Skelet, wenn vorhanden, nur aus isolirten Sternchen, resp. Kugelsternchen bestehend. Kanalsystem nach dem dritten oder vierten Typus.

Fa m. Ha lis a r c id a e.

Kein Skelet. Keine Faserrinde. Grundsubstanz iiberall hyalin. Oberfäche glatt, sammetartig oder schliipfrig.

$$
\text { Ginus: Halisarca. D u j a } \mathrm{d} \text { in. }
$$

Kanalsystem nach dem dritten Typus. Geisselliammern läng. lich, oft veristelt Mittelmeer (Adriatisches Meer, Neapcl), Atlantischer Occan, Ostsee.

(1ig. 27.) 9. Spec.: Halisarea Dujardini. J o hn s to n.

Hal. Dnjardini vertritt in der Ostsec die Sarkospongien. Die Entecknng der Sarkospongien verdanken wir D u jar d i ${ }^{54}$, der I 828 den ersten Weichschwamm beschrieb; er gab ihm jedoch keinen Namen. Johnston ${ }^{55}$ ), der denselben Schwamm an der britischen Kiiste beobachtete, führte ihn unter dem Namen Hal. Drijardini in das System cin. Eine nahere Beschrcibung giebt damn Li e berkith $\left.n^{56}\right)$. F. E. Schulze ${ }^{57}$ ) hat den Bau von Hal. Dujardini eingehend studirt, sowohl an Exemplaren des Mittelmeers, als auch an solchen der Ostsee. Da ich bej meinen Untersuchungen an Hal. Dujardini nicht $\%$ abweichenden Resultaten gekommen bin, so verweise ich auf dic Arbeit F. L. Schulze's.

5) F, D ujardin. Observations sur les Lenges et en particulier sur la Sipongille ou liponge d'eau donce. Amales des Sciences nat. Serie II. Tom. ro. I8.38. pag. 6

5ii) J o h n sto $n$, Brit. Sponges. pag. 192 .

si) L ieberkith n, Beitr. zur Anatomie d. Spongien. Archiv für An. u. l'hys. Is 59 . S. $353-357$.

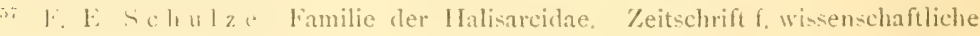
Zaologie, Br. $2 S$ is 577 S. 1 . 
Subordo: Clavulina.

Schwämme von ziemlich fester Konsisten\%. Line Faserrinde oft vorhanden. Bei den nicht ganz unregelmässig geformten ist cine radiäre Struktur noch in Slelet sichtbar. Charaliteristisch sind die meist ausgeprägt geknöpften Spikula. Kanalsystem nach dem vicrten, sclegentlich nach dem dritten Typus.

\section{Iiam. Polymastidac.}

Skeletelenente radiä gelagert. Fiascrinde meist sclar eleutlich. Auf der Oberfläehe Warzen oder Papillen ron verschiedener Gestalt. Kamalsystem nach den vierten Typus.

(ienus: Polymastic. Bow erbank.

IIeist flach scheiben- oder liugelsegmentformigy. Dic cine (Oberfliche mit kiureren oder längeren, oft (nicht immer) als Oskularoln fungirenden Anhängen. Faserrinde deutlich. Spic. indic, tr."ac. und trac, mit Variationen und von schr verschiedener Grösse. Arktischer Ocean (Küste von Norwegen; Barents-1leer : Nittelmeer (Neapel, Adria). 2-165 Faden. Auf Steinen.

(Fiig. 28.) 1(). Spece: Polymastia mamillaris. Bow er bat $n$ k.

(Rinalde arctica. Me rejkowsky.)

(Polymastia penicillus. Vosmacr.)

Das Genus Polimastia wurde I 864 von Bow $\mathrm{crbank} \mathrm{k}^{5 \mathrm{~s}}$ ) mit folgenden Merkmalen aufgestellt: Skcleton. Basal mass. Central portion consisting of a plexus of contorted anastomosing fasciculi, resolving themsclves near the surface into short straight bundles disposed at nearly right angles to the surface. Oscula congregated, clevated on numerous long fistulae. Fistulac composed of numerous parallel fasciculi, radiating from the base to the apex of each in straight or slightly spiral lines." As Typus des Genus nennt er Pol. mamillaris, die er identifizirt mit Spongria penicillus Non ta gu 


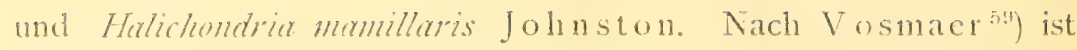
jedoch Pol. mamillaris Bowerbank mit Herlehondria mamillaris Jonston nicht identisch, wohl aber Merejkowsky's ${ }^{60}$ ) Kimaled arctice und Pol. penicillus Vosmaer. Schmidt's "il) Genus Rinalda is 70 von ihm aufgestellt als Genus, das Uebergangsformen von den Subcriten zu den Tethyen umfasst, ist, wic schon Vosmaer $\left.{ }^{62}\right)$ gezeigt hat, mit Bowerbank's Polymastia identisch.

An einem ausgewachsenen lixemplar von Pol. mamillaris muss man zwischen dem Basalstiuck und den auf demselben sich er. hebenden Papillen unterscheiden. Das Basalstick ist von lester Konsistenz, 4-5 cm breit und $3 \mathrm{~cm}$ hoch. Die Papillen erreichen cine Höhe von $8 \mathrm{~cm}$. Sie stehen zicmlich dicht. Ihre Dicke beträgt $4-5 \mathrm{~mm}$; nach oben $z u$ findet eine Verjüngung statt. Charakteristisch für Pol. mamillaris ist die Anordnung der Kieselnadeln. Vom centralen Teil des Schwammes aus strahlen, senkiccht nach der Oberfäche hin verlaufend, Bündel von Nadeln, Fascikcl genannt. Nach dem Mittelpunkt $z u$ sind die I'ascikel $0,14 \mathrm{~mm}$ dick; nach der Peripherie zu werden sic dicker, und der Durchmesser eines Bindels beträgt hier $0,5 \mathrm{~mm}$. Im centralen Teil vereinigen sie sich zu einem Geflecht. Die Nadeln, aus denen sie bestehen, sind gestreckt; entweder in beiden Enden abgerundet, oder an eincm Ende abgerundet und am anderen Ende spitz. (Fig. 29 a. b. c.) Die an beiden Enden abgerundeten Nadeln sind $0,588 \mathrm{~mm}$ lang; die Dicke beträgt an dem einen Ende $0,028 \mathrm{~mm}$, am anderen $0,02 \mathrm{mn}$. Dic an cinem Encle abgerundeten, am anderen Iinde spitzen Nadeln sind in der Mitte bauchig verdickt, I, I2- I, $26 \mathrm{~mm}$ lang, o,o $18 \mathrm{~mm}$ dick. Häufig zeigen dic Nadeln am stumpfen Ende eine Einbuchtung, sodass sie das Aussehen der Nadeln von Dosmacidon arciferm Schmidt haben. In den Papillen oder Röhren verlaufen ebenfalls Nadelbiundel. Schon bei makrosliopischer

an Vosm a er, Willem Larent's Expedition. Niederländ. Archiv f. Zuoluric, Keport on the sponges dredged up in the Arctic Sea by the Willem Barents. 1878 u. 79. Suppl. I. IS8I-ISS2, S. $26-30$.

in Merejkowsky, Ltudes sur les leponges de la Mer Blancluc. Mem. de l'Acar, Imp. des Sciences de Sit, l'etersbg. 'T. 26. 7. 1879. S. 4-I5.

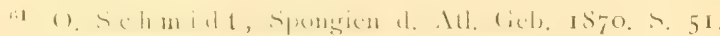

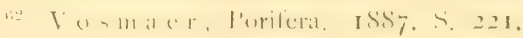


Betrachtung benerkt man, senkecht vom (irnnde der Papille sich crhebend, iber $1 / 1 \mathrm{~mm}$ dicke mit cinander in geringen Mbstinden parallel in einer Spirale bis an die Spitze der Tapille verlatiende Bindel. Durchkreuzt werden sic von ringförmig angeordneten, diunneren Nadelbiindeln, sodass ein Netzwerk von riemlich quadratischen Maschen entsteht. Die Nadeln in den ringfömigen Bündeln haben diesclbe Form, wic die Nadeln in den Fasciculi, nur sind sic etwas kleiner, 0.7-1.0 mm lang. Die quadratischen Maschen enthalten cbenfalls Nadeln, welche strahlig oder büschelförmig angeordnet sind und grösstenteils die Form von Stecknadeln haben: sic sind seschweift oder gebogen, 0,2 I mm lang. (Fig. 29 d.) Dazwischen finden sich auch vereinzelt Nadeln, wic sic in den Fasern von Chalina limbater vorkommen, spitz-spitz, 0,098 mm lang. Sowohl in den Bündeln der Papillen als auch in den Maschen habe ich häufig lieselige Gebilde von mehr oder weniger regelmässigg kugeliger oder ciformiger Gestalt gefunden; sie sind im Durchmesser $0,056-0,084 \mathrm{~mm}$ dick. Pol. mamillaris besitzt cine deutlich ausgeprïgte Rinde von $0,5 \mathrm{~mm}$ Dicke, die an Spirituspracparaten weis. gefurbt ist, während das Innenparenchym grau erscheint.

Ein liktoderm in Gestalt eines Plattenepithels habe ich nicht nachweiscn können, da mir nur ein Spirituspraeparat zur Verfugung stand. Die Rincle zeigt eine faserige Struktur olnne ausgeprägte zellige Elemente. Dic Grundsubstanz des Innenparenchyms ist könchenreich; in ihr eingelagert sind spindelförmige Zellen und amocboide Wanderzellen von $0,008 \mathrm{~mm}$ Grösse. Das Kanalsystem ist nach dem vicrten Typus gebaut. Sowohl dic zufuilurenden, als auch die abführenden Kanale sind reich verzweigt.

\section{Porifera calcarea.}

Dats immer vorhandene Slielet besteht aus Spikula von kohlensaurem Kalk. Kanalsystem nach dem ersten, zweiten, dritten oder vicrten Typus sebildet.

$$
\text { () r clo: Homococla. }
$$

Besondere (icisselkammern fehlen; statt ihrer ist fast die ganze innere Höle mit Kragenepithel ausgekleidet. Kanalsystem also nach dem crsten Typus. 
Fam. $\lambda$ iseon idac.

Dic einzige Familic hat die Charaktere der Orelnmms.

Cemms Lemeosolemia. Bow e r b a $n \mathrm{k}$.

Dic einzige Gattung hat die Charaktere der Ordnung. Atl. Pacif, uncl Inclischer Occan. Mittelmeer. Unticf.

11. Sper.: Ience coriaced. Bow e r b a n k.

Dic ersten Angaben uber diesen Schwamm finden wir nach 11 a e c ke $1^{63}$ ) zuerst bei $\mathrm{M}$ o $\left.n \mathrm{ta} \mathrm{gu}^{64}\right)$, der ihn Spongia coriarea und bei Johnsto $\left.{ }^{(5 i}\right)$, der ihn Grantia coriacea nannte. I 886 wurcle er von $\mathrm{B}$ o w e r b a $\mathrm{n} \mathrm{k}^{-6}$ ) in clas System eingefülurt: Sponge. Sessile incrusting; fistulae tortuous, anastomosing; surface smooth, parities thin. Cloacal cavity continuous, unarmed internally; mouths inconspicuous. Oscula and pores inconspicuous. Spicula of skeleton equiangular triradiate, radii thick, apices obtusely printed. Mundöfnumgen hat Bowerbank nicht bemerken können. Nach $\mathrm{H}$ a e ck elii), der Lene. coriacea als Ascetta coriacen ausfuihrlich beschreibt, hat Bow erban $\mathrm{k}$ nur die mundlose Stockform (Auloplegma) zur Untersuchung gehabt. Ich habe ebenfalls keine Mundöffnungen an den mir zur Untersuchung vorliegenden Exemplaren, die kleine formlose Massen von 2 mm Grösse dar. stellten, finden können. Ielucosolemia coriacea tritt nach $\mathrm{Hacckel}$ unter I I verschicdenen Formen auf: »als Olynthus, Olynthella, Cistolynthus, Soleniscus, Solenula, Nardorus, Nardopsis, Tarrus, Tarropsis, Auloplegma, Ascometra. Sie verdient desshalb wegren der ausserordentlichen Mannigfaltigkeit ihrer äusseren Erscheinung cine besondere Beachtung. " (Fig. 30.) Die Nadeln sind reguläre Dreistrahler. Dic einzelnen Strahlen $0,06 \mathrm{~mm}$ lang.

\footnotetext{
(i3) If a ecke 1, Mlonographie der Kalkschwämme. II. \$, 26.

i4) Nontagu, Wernerian Memoirs. p. I 16.

(ii) J o h nst on, British Sponges and I itophytes, p. I83

136) Bowerbank, A Monograph of British Spongiadae. Vol. 11 p. 34.

iz Il a e ckel, Monographie der Kalkschwämme, II. S. 24-;0
} 


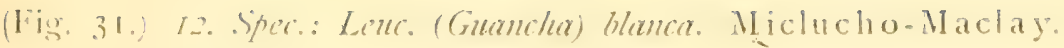
(.1scotta blance. Hackel.)

Dicser Kalkschwamm wurde zuerst von Miclucho. Maclay tis beschricben, der ihm bei den Kanarischen Inseln, sowohl einzelne l'crsonen, als auch verzweigte Stöclichen bildend, fand. Haeckel ${ }^{69}$ ) beschreibt denselben Schwamm als Ascetta blanea nach Formen von den Philippinen. In der Kieler Bucht bildet Leus. blanea cinzclne gesticlte Personen auf Algen von $2 \mathrm{~mm}$ Linge und $0,3 \mathrm{~mm}$ Dicke. Dic Nadeln sind Dreistrahler. Der eine Strahl, Basalstrahl genannt, lainger als die beiden anderen Lateralstrahlen. Bei den Formen der Kanarischen Inseln ist der Basalstrahl $0,1--0,2 \mathrm{~mm}$ lang, der Lateralstrahl 0,05-0,06 mm lang; bei denen der Philippinen ist cler Basalstrahl 0,OS-O, I mm, der Lateralstrahl 0,05 mm lang. Bei den Ostscecxemplaren ist der Basalstrahl 0,72, der Lateralstrahl $0,36 \mathrm{~mm}$ lang. (Fig. 32.)

(lig. 33.) 13. Spec.: Lente. (Ascetta) sagittariar. II a c ckel.

Dieser Schwamm wurde 1872 von Hackelio) unter dem Namen Ascetta sagittaria in das System eingefulurt. Er wurde auf der l'ommeraniacxpedition im Sommer i87 I von Mocbius im grossen belt gesammelt. Lis sind einzelne Personen auf Sertularia, $2-5 \mathrm{~mm}$ lang und $\mathrm{I}--2 \mathrm{~mm}$ dick. Die Mundoffnung $0,8-\mathrm{I}, 0 \mathrm{~mm}$ gross. Die Nadeln sind Dreistrahler, pfeilformig. (Fig. 34.) Der Basalstrahl ist $0, \mathrm{I}-\mathrm{O}, \mathrm{I} 2 \mathrm{~mm}$ lang und nach abwärts gerichtet. Dic Lateralstrahien, 0,05-- 0,06 $\mathrm{mm}$ lang, sind nach oben gerichtet und bilden einen konkaven Bozen.

(lig. 35.) 13. Spec: Lenc. botryoides. James-Clark. (Grantia Liebrokiuluii. Schmidt.)

(Ascortis fragilis. Hacckel.)

Nach Haeckelil) wird dieser Schwamm zuerst von J a mesClark ${ }^{22}$ ) beschrieben als buschförmiges Stöckchen, $30-40 \mathrm{~mm} \mathrm{im}$

(ix) M i cluch o- Ma cla y. Beiträige zur Kenntniss der Spongien. I. Jenaische Zeitschrift. IV. I\$6S. S. 221 .

(in) II a eckel, Monographie der kalkschwimme. Jid. Il. S. 35-40,

ii, H a eckel, Monographic der Kalkschwämme, II, S. \&2.

i1) Ha ecke1, Monorraphie der kalkschwämme. 11. S. 7.t.

i2, Memoirs boston Soc. Vol, 1. P't. 3. p. I9. 
Durchmesser an den Küsten der Vereinigten Staaten. Die Nadein sind unregelmässige Dreistrahler und einfache Stabnadeln. Eine ausfürliche Beschreibung giebt dann Ha eckel selbst: Die liorm, sagt $\mathrm{C}$, die jeh an der Küste von Norwegen (in der Nähe von Bergen) gefunden habe, stellte im geschlechtsreifen Zustande teils isolirte nacktmündige Personen dar (Olynthus), teils kleine Stöcke mit lauter nacktmündigen Personen (Soleniscus). l.etztere Form zeigen auch die von Moebius in der Ostsee (gr. Belt) gesammelten Stöckchen. Die Einzelpersonen sind unregelmässig länglich, rund oder ciformig, kurz gestielt, $1,0-1,5 \mathrm{~mm}$ lang, $0,3-0,5 \mathrm{~mm}$ dick. Die einfache nackte Mundöffnung ist $0,2 \mathrm{~mm}$ breit. Die kleinen Stöckchen haben 5-10 mm Durchmesser, bilden teils kleine buschförmige Klumpen auf Algen, teils bilden sie an der Unterfläche von Steinen cin dünnes in einer Ebene ausgebreitetes Geflecht anastomosirender Röhren von $0,5-1,5 \mathrm{~mm}$ Länge und $0,2-0,3 \mathrm{~mm}$ Dicke.

Die fiormen aus dem zoologischen Museum in Kiel, und auch diejenigen, die ich in der Kieler Bucht fand, bilden kleine Stöckchen, deren Einzelpersonen $46 \mathrm{~mm}$ lang sind, also bedeutend grosser, als wie Hackel sie beschreibt. In »Neue Beiträge zur Anatomic der Spongien «, beschreibt Lieberküh $n^{73}$ ) einen Kalkschwamm, den er bei Triest und Helgoland gefunden, und den er bei den damals bekannten Species nicht unterbringen lionnte. Er sagt: "In mancher Beziehung stimmt er mit Grantia botryoides überein, abor in dem Verhalten der Nadeln weicht er ab. « Dic englische Form hat nur dreistrahlige Nadeln, während der Lieberkühn'sche Schwamm neben den Dreistrahlern noch einfache Stabnadeln besitzt. Lieberkühn macht keine neue Species daraus. Schmidt ${ }^{74}$ ) giebt ihm den Namen Grantia Lieberkiilnii Schmidt. Ich glaube, dass Schmidt's Grantia Lieberkïhnii identisch ist mit JamesClark's Lencosolenia botryoides und Haeckel's Ascortis fragilis.

Die Dreistrahler sind unregelmässig; ungleichwinklig. Dic cinzelnen Strahlen sind verschieden lang (0,09-0, $22 \mathrm{~mm})$. (Fig. 36.) Die Stabnadeln haben eine Länge von $0,1-0,18 \mathrm{~mm}$.

78) L i eberk tihn, Neue Beiträgre an Anatomic der Spongien. Arehiv $f$. An, u. Phys, 1S59, S. 373 u. 382 .

i4) Schmidt, die Spongien des Adriatischen Mecres, IS62. S. 17. 


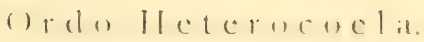

Geisselkammern vorhanden. Die nicht Kragenzellen tragenden Teile sind mit cinfachen lipithel ausgekleidet. Kanalsystem nach dem zuciten, dritten oder vierten Typus.

$$
\text { liam. Syconidac. }
$$

Dic srossen länglichen Geisselkammen (Radialtuben) stehen radiair mm dic Kloakal-Höhle und mïnden direkt hincin.

$$
\text { Gemlls: Sycon Risso. }
$$

Mit den Charalitiren der Familie.

(Fig. 37.) 5.5. Spece: Syeon ciliatum L i c berk iuhn.

(Sicandra ciliato $\mathrm{H}$ a e c k e I).

L, i eberkïhn, "5) der diesen Kalkschwamm bei Triest und Helgoland fand, beschreibt ihn folgendermassen: „Dic grösstcn Exemplare hatten fast die Länge von einem Zoll und waren 3-4 Linien dick. In ihrer Gestalt gleichen sic einander vollständig, und ist dieselbe so wenig schwankend, wie sonst bei licinem der bekannten Schwamme. Sie sind drehrund und nehmen nach der Ansatzstelle zu erheblich und an dem oberen linde hin etwas an Durchmesser ab. Am oberen Ende findet sich ein fast wic Asbest glänzender Kranz von Nadeln, welche weit iber das Körperparenchym hinausragen.

Ha c k elii) beschreibt inn als Syandra riliata. Die Nadeln sind Dreistrahler. Vierstrahler und Stabnadeln. Die Strahlen der Dreistrahler sind $0,1-0,2 \mathrm{~mm}$, die der Vierstrahler $0,15-0,25 \mathrm{~mm}$, dic Stabnadeln $\mathrm{I}-3 \mathrm{~mm}$ lang.

$\therefore$ l. i e be $r$ ki h $\|$, beiträge yur Anatomic der Spongien. Archiv f. Anah 11. I'hys. 1454. ‥ 37.

IS65. S. 737 .

-. Beiträge zur Anatomic d. Kalkspongien. Archiv f. Anat. U. Mỵs.

II a ecke 1, Monographic der kalkschwimme. 1. S. 206--303. 
Die hauptsächlichsten Nerkmale der

\section{Species}

Matichondriar panica Pall.

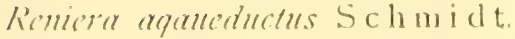

Ren. (Isodyctia) itarians S S h m id t.

Chatimula orulum Schmidt.

Chalimula fertilis heller.

Chalina limbata Bow er bank.

Parhychalina rustica Sch midt.

Esperella (Esperia) lanugo Schmidt.

Halisarea Dujardini J o h n ston.

Polymastia manillaris 13 ow e rbank.

Lencosolenia coriacea Bow e rbank.

\section{Gestalt}

Unregelmässig, lappig od. rundlich ciformig.

Meist rohrenformig, selten unregelmässig.

lingerförmig verzweigt.

Rundlich, cllipsoidisch.

Röhrenfömig, oben verdickt.

Eine od. mehrere köhren bildend.

Röhrenförmig, nach oben zu keulenförmig verdickt.

Rundlich.

Rundlich, länglich, scheibenformig. Gewölbtes Basalstiick mit $8 \mathrm{~cm}$ hohen Papillen.

Stöckchenbildend 
in der Ostsee rorkommenden Spongien:

\section{Farbe}

sselblich oder grimlich.

bläulich oder rötlich, seltener wcisslich.

gell)lich.

in sporitus bräunlich

in Spiritus gelblich. selblich oder bräunlich.

in Spiritus braumgelb.

in Spiritus bräunlich.

weiss oder gelblich. in Spiritus weiss.

weiss.

\section{Skelet}

Aus Kieselnadeln bestehend von unregelmässiger Anordnung. Die Nadeln sind an beiden linden spitz. Amphitorne und Amphioxe. Daneben Amphistrongyle, Tylostyle und ()xydiactine. 0,36 mm lang. 0,01 $\mathrm{mm}$ dick.

Nadeln an den Spitzen durch Spongin verbunden, ein Net\%werk von ziemlich quadratischen Maschen bildend. Amphitorne. 0,12-0.16 mm lang.

Nadeln an der Oberfläche durch Spongin zu eincm Netzwerl verbunden; in älteren Teilen in Sponginfasem cingebettet. Amphitorne. O, I mm lang. Nadeln in schwach ausgebildete Sponginfasern eingebettet, die pinselförmig uiber die Oberfläche hervorragen. $0,176 \mathrm{~mm}$ lang.

Nadeln in Sponginfasern. 0,098 mm lang.

Nadeln in Sponginfasern, die stark ausgebildet sind, eingebettet. $0,098 \mathrm{~mm}$ lang. Das Sponginfasergeriist aus Kreis-, I.ings- und Radiarfasern bestehcnd.

Sponginfasern stark ausgebildet, unreschmissig verlaufend. Nadeln $0,08 \mathrm{~mm}$ lang. Amphioxic und Oxydiactine.

Nadeln durch Spongin verkittet, am cinen linde spitz, am anderen linde abgerundet, $0.22 \mathrm{~mm}$ lang. Daneben Haken (Schiufeln, dic nach beiden Enden hin völlig gleich entwickelt sind. ()hne Skelet.

Skeletelemente radiär gelagert. Charakterisch sind dic Stecknadeln. Tylostyle.

Skelet aus Kallinadeln bestehend. Regulire 1)reistrahler. 


\section{Species}

Lencosolenia (Ascetta) sagittaria Ha eckel.

Leucosolenia (Guanha) blanca Miklucho-M aclay.

Lincosolentar botryoldes JamesClark.

Sycon ciliatum Li e berk ï bn.

\section{Gestalt}

Einzelne gestielte Personen $2--5-\mathrm{mm}$ lang, $\mathrm{I}--2 \mathrm{~mm}$ dick.

Einzelne gestielte l'ersonen, $2 \mathrm{~mm}$ lang, $0,3 \mathrm{~mm}$ breit.

Buschförmige Stöckchen mit $4-6 \mathrm{mmm}$ langen Personen.

Kegelförmig mit einem Kranz von Nadeln in der Umgebung der Mundöffnung. 


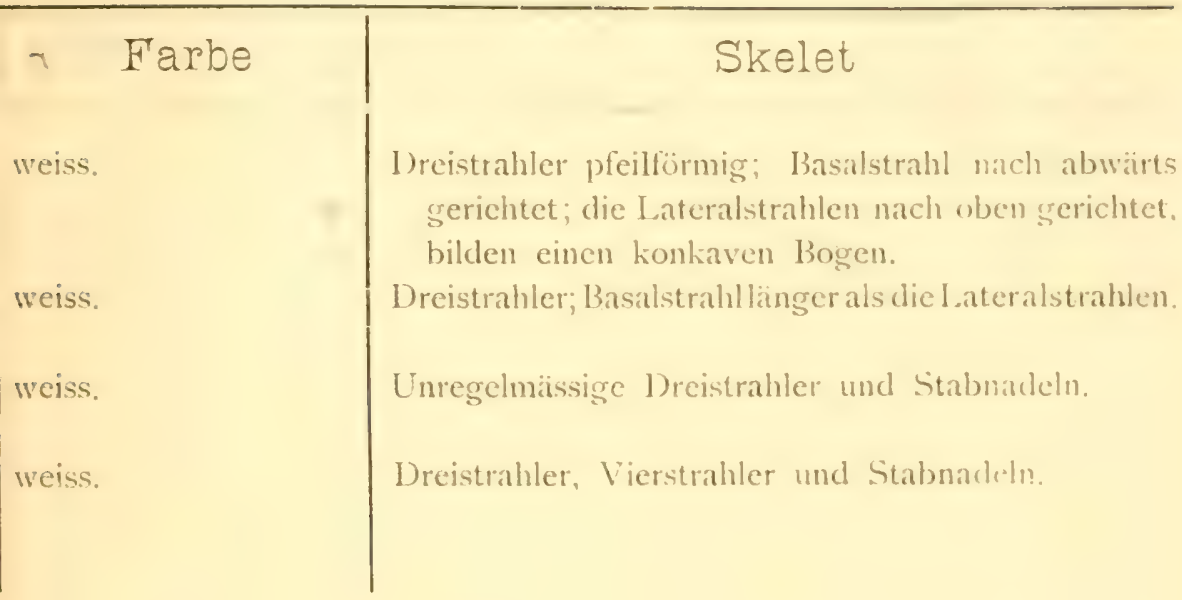




\section{Besti in III II II g'}

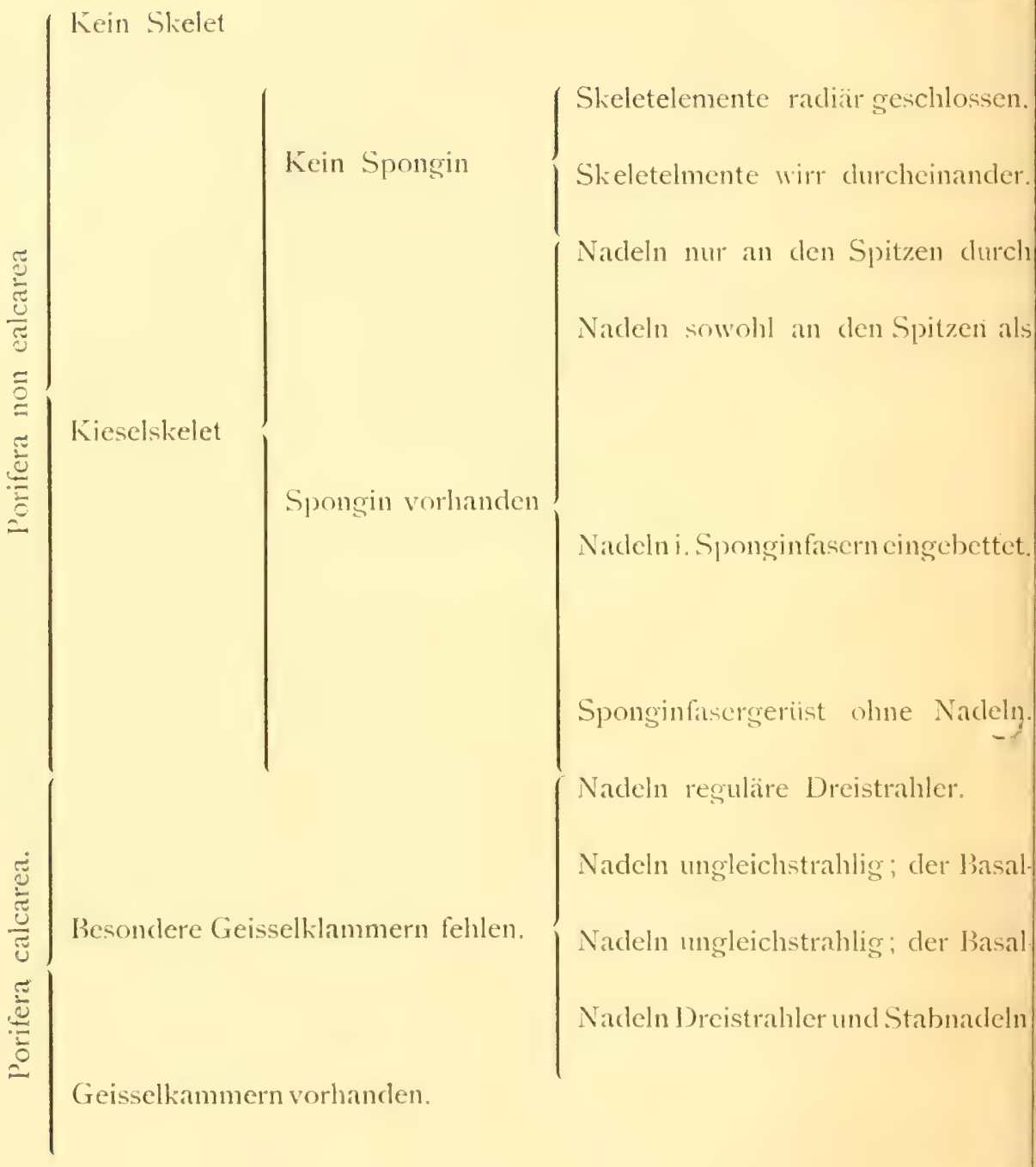




\section{Tabelle.}

Hal. Injardini

Johnston.

I')l. mamillaris

Bow erbank.

llerl. panicere

P'a 11.

Spongin verbunden.

Limiera anneductus

$\therefore \mathrm{c} / \mathrm{m} \mathrm{mi} \mathrm{d}$.

auch der I änge nach durch Spongin verbunden. Remere (Lsodjetia) ararians

Sponginfasern schwach $\int \begin{aligned} & \text { Schwamm rundlich. } \\ & \text { (Nadeln } 0,17 \mathrm{~mm} \mathrm{1.)}\end{aligned}$

B ow e r b a n k. auscrebilclet.

CHalimula ormlum

$i 11$

Schwamm röhenfömig. Chalimula fertilis

(Naclein 0,098 mm l.) Keller.

Sponginlasern stark

regemässig angeordnct. Chalina limbater

atusgebildet.

unregelm. angeordnet. P'achechalina rustica

Siclin ind

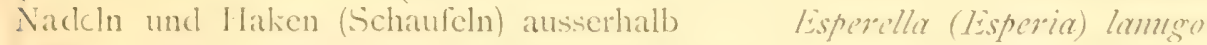

desselben liegend.

$\mathrm{Schmi}$ i t

Lencosolenia coriacea

B ow er ba nk.

strahl länger als dic Iatcralstrahlen, dic einen Lencosol. (Guancha) blanca

spitzen Winkel bilden. Miklucho-Maclay.

strahl nach abwärts gerichtet; dic Lateralstrahlen Lencosol. (Ascetter) sorgitforiu bilden einen konkaven Bogen.

Lencosolemia botroyoides

James-Clark.

Sycon iliatum

L, ieberliihn. 


\section{Litteratnr-Verzeichniss.}

1. M. Brau n, Physikalische und biologische Untersuchungen im westlichen Teile des finnischen Meerbusens. Archiv fiir die Naturkunde Liv-, Esth- und Kurlands. Serie II. Bd. IO.

2. - - Faunistische Uutersuchungen der Bucht von Wismar Archiv der Freunde fuir Naturgeschichte in Mecklenburg. Jahrgang 42. 1888 .

3. Bowerbank, A, Monograph of British Spongiadae. Vol. I-IV. London, Ray Society I $\$ 64,66,74,82$

+ James Clark, On the Spongiae ciliatae as Infusoria flagellata or observations on Leucosolenia botryoides in $\mathrm{Ann}$. and Mag. I. I 868 .

5. Dujardin, Observations sur les Eponges. In Ann. Sc. nat. X. $183 \hat{s}$

6. Ha eckel, Monograplie der Kalkschwämme. 1872 .

7. Jahresbericht der Kommission zur wissenschaftlichen Untersuchung der deutschen Meere fuir das Jahr I\$7 I.

$\therefore$ _ - dasselbe fuir die Jahre $1 \$ 72$ und 1873.

y. Ke!ler. Ueber den Bau von Kemiera semitululosa $\mathrm{S} \mathrm{ch} \mathrm{m} \mathrm{i} \mathrm{t}$. Zeitschrift für wissenschaftliche Zoologie. Brl. 30. I 878.

10 - - Studien uber Organisation und Entwicklung der Chali. ne?. Zeitschr. für wissenschaftliche /oologic. B3d. 33. 1879.

I I. - - Die Spongienfaum des roten Meercs. \%citschrift fiir wissenschaftliche Zoologie. Bd. 48. I889.

I2. Johnston, History of British Sponges and Lithophytes. Einburgh 1842 .

1.;. Von Lendenfeld. The vestibule of Dendrilla caremosa The Proc of the Linn. Soc. of New. South Wales. Vol. X. I885.

1.4. - Synocil's Simnesorgane d. Spongien. \%ool. Anz. I887

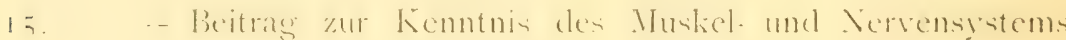
er Hornspongien. Sitzungsbericht d. Kgl. Preuss. Akad. 1. Wissenschalten. I 885 . Teil II.

10 - - Experimentelle Untersuchungen ïber dic Physiologric der Spongien. \%eitschrift für wissenschaftliche Zoologie. lid. 48. 1850 . 
1;. I. I.endenfeld, Die (Juline'll des australischen Gebiets. Zoologische Jahrbiicher. I887.

1.. - Notiz über den Bat der Geisselkammern der Sipongien. Zoologischer Anzeiger. Bd. 12. 1889.

(1). Lenz, Dic wirbellosen Tiere der Travemuinder Bucht. Teil I. Anhang zu dem Jahresberichte der Kommission zu Lntersuchung der deutschen Mecre. 1874 und 1875.

$\therefore$ I. ic berkïhn, Beiträge zur Anatomie der Spongien. Archiv fiir Anatomic und Physiologic. I 857.

$\therefore \quad$ - Nene Beiträge zur Anatomie der Spongicn. Archiv fur Anatonic und Physiologic. I 859.

2 - - Beitrage zur Anatomie der Kallspongien. Archiv fü Anatomic und Physiologic. I 865.

ㄱ. Martens, Reise nach Venedig. II. Ulm 1824.

24. Merejkowsky, Etudes sur les Iiponges de la Mer Blanche. In Mém. Icad. St. Pétersbourg. XXVI. 7. I\$79.

$\therefore$. Miklucho-Maclay, Beitrage zur henntuiss der Spongien. I. In Jenaische Zcitschrift. IV. IS64.

zi. Montagu, lissay on Sponges. In Mem. Wern. Soc. II 1. 1 sis.

27. Nardo, Spongiariorum Classificatio. In Isis (Olien). I\$33.

2i. Noll, Beiträge zur Naturgeschichte der Kiesclschwämme. I. Desmacidon bosei Noll mit Hinweisen auf Crandellar carmosa Kiippel and Spongilla fragilis. Frankfurt a. Main. 1888.

2). O. Schmidt, Die Spongien des Adriatischen Mecres. I 862. 30. - I. Supplement der Spongrien des Adriatischen Mecres. $1 \$ 64$.

i. - -. II. Supplement der Spongien des Adriatischen Mecres. 1860.

i2. - - Dje Spongien der Küste von Algier (III. Supplement der Spongien des Adriatischen Meeres). I 868 .

i. - - Grundziige einer Spongienfauna des Atlantischen Gebietes. 1870 .

i.t. Ii. If. Schul\%e, Ueber den bau und dic Entwicklung von Syeandrer raphamus. Zeitschrift für wissenschaftliche \%oologic. XXV. Supplement. 1875.

$\therefore$ - - Untersuchungen iber den Bau und die lintwicklung der Spongien. II. Herliseren Zeitschr. f. wiss. Zool. XXVIII. IS77. 
.3'. IX. Dic Familic der Aplysinidac. Zeitschr. f. Wiss. Zool. XXX. I 878 .

37. - - VII. Dic Familic der Spongidac, Zeitschr. f. wiss. Zool. XXXII. 1879 .

is. - - IX. Dic Plakiniden. Zcitschr. f. wiss, Zool. XXXIV. 1880 .

3). - - und Lendenfeld, Ueber die liczeichnung der Spongiennadeln. Berlin I 889 .

fo. W. J. Sollas, Tetractincllida. Reports on the Scientific Results of the voyage of $H . M$. S. Challenger. Loology. Iol. XXV. 1). IVI

+1. Vosmaer, Bronn's Klassen und Ordnungen der Spongien (Porifera). I 887.

4. - - Report on the Sponges dredged up in the Aretic Sea by the Wilkelm Barents" in the years I 878 and 1879 . In Niederl. Archiv für hool. Suppl. I. (1882) pag. I-73.

\section{Hinlïuterung der Figuren.}

Figg I. Halichondria panicer P'all, aus der Kieler Bucht. Natiurliche Grösse.

. 2. Hal. panicea Pall, gefunden zwischen Fehmaru und Langeland. Natïliche Grösse.

.. 3. a.-k. Nadeln von Hal. panicea Pa ll. a. b. d. e. f. g. h. k. 200 fach, c. 300 fach, i. 250 fach vetgrössert.

.4. Hal. panicea. Pa 11, Plattenepithel. 745 fach verourössert.

. 5. , , , Geisselkammer, von oben gesehen. 300 fach vergr.

. 6. " , , Geisselkammer, Querschnitt. 300 fach vergi.

, 7. " , , a. Sternförmige Bindegewebszellcn (Ganglienzellen?) c. Eizelle, 745 fach vergr. b. Contraktile liascrzellen, 745 fach vergr, 


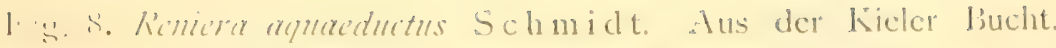
Natiirliche Grösse.

Anordnung der Nadeln. 200 f. vergr.

.. 10. " " Silikoblasten. 450 fach vergr.

.. I1. Remuer (Isodyctia) suriens Bowerbank. Aus der Kicler Bucht. Nat. Grösse.

. I 2. " " Nadeln. a. 350 f. b. 400 f. vergr. .. 1. Chalimula orulum Schmidt. Aus der Kieler Bucht. Nat. Grösse.

. 1. ., .. Nadeln. 200 fach vergr.

.. 15. Chalimula fortilis Keller. Aus der Kieler Bucht. Nat. Grösse.

.. 19. Chalima limbata Bow erbank. Aus der Kieler Bucht. Nat. Grösse.

. 1, " " "Sponginfaser. 1501. vergr.

. IS. . "Nadeln. I50 f, vergr.

.. r. ," Spongoblasten. 745 f. vergr.

.2 20. Parhychalina ronstior Schmidt. Aus der Kieler bucht. Nat. Grösse.

"21. " " Oxydiactine. 150 f. vergr.

.. 2. Lisperelle (Isperia) lamugo $\mathrm{Schmidt}$. Aus dem Gr. Belt. Nat. Grösse.

" $2 . \quad$ " , . Haken (Schaufelnj. 745 f. vergr. "2f " " " Sponginfasergeriist. In den Maschen sieht man Zellenreste. $120 \mathrm{f}$. vergr.

. $2 . \quad$. . . Einzelne isolirte Zellen, dic die Sponginfaser ausschciden. $745 \mathrm{f}$. verg.

.. 2r. . . " Einige Kreisfasern vor ihrer Vereinigung, $200 \mathrm{f}$, vergr.

"2.- Talisara Dujardini Johnston. Aus der Kieler Bucht. Nat. Grösse.

.. 2. Polymastie mumillaris Bowerbank. Aus dem Gr. Belt. Nat. Grösse.

. 29. a-d .. "Nadeln. a 100 f. b gof. c gof. d. I 50 f. vergr. 


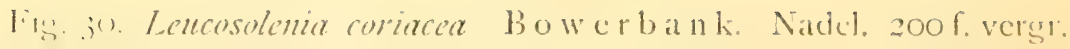

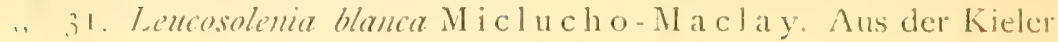
Nat. Grössc.

. 3.2 " " Nadel. 20o fo vergr.

. 3.i. Leneosolenia (Ascelta) sagittaria $\mathrm{H}$ a e ckel. Aus der Kieler Bucht, 2 f. vergr.

. 31. " " " Nadel. 200f. vergr.

.. 35. Lemesolenier botryoides James-Clark. Aus der Kieler Bucht. Nat. Grösse.

.. 3i. " " N Nadel. $200 \mathrm{f}$, vergr.

.. 3\%. Sycon riliatum Lieberkübn. Aus der Kieler Bucht. Nat. Grösse.

.. i. " , N Nadeln. Ioo f. vergr. 


\section{Tubenslanf:}

Geboren bin ich Max Theodor Grentzenberg am 20. Janual i 865 7u Danzig als Solnn des daselbst verstorbenen Kaufmanns Albert Grentzenberg. In meiner Vaterstadt besuchte ich das Realgymnasium zu St. Petri und Pauli, das ich Ostern I 886 mit dem \%eugnis der Reife verliess, um zunächst sechs Semester in Berlin, dann in Kiel Naturwissenschaften zu studieren.

Vorlesungen hörte ich bei folgenden Herren Professoren und Docenten :

In Berlin: Beyrich, Du Bois Reymond, Heider, v. Helmholtz, Hettner, Klein, Korschelt, Krabbe, Landolt, v. Martens, l'aulsen, F. L. Schulze, Schwendener, Secliger, Sell, Waldeyer.

In Kiel: Brandt, Curtius, Deussen, Glogau, Karsten, Krimmel, Reinke, Schiitt, Tönnies, Weber.

Allen diesen meinen Herren sage meinen besten Dank. \% / besonderem Danke fihle ich mich Herrn Prof. K. Brandt und Herm Privatdocenten 1)r. Fried. Dahl verpflichtet, die mich bei der Anfertigung der Arbeit mit Rat und That unterstuitaten. 


\section{Thesen.}

I.

Bei allon Metazoen finden sich Nervenelemente.

II

Die Kontinuität der Lebensvorgange kann nicht völlig auf längere \%eit ohne Gefährdung des Lebens unterbrochen werden.

III.

Die Wanderung der Organismen und ihre Isolirung ist eine vorteilhalte Bedingung fiir die lintstehung nener Arten. 



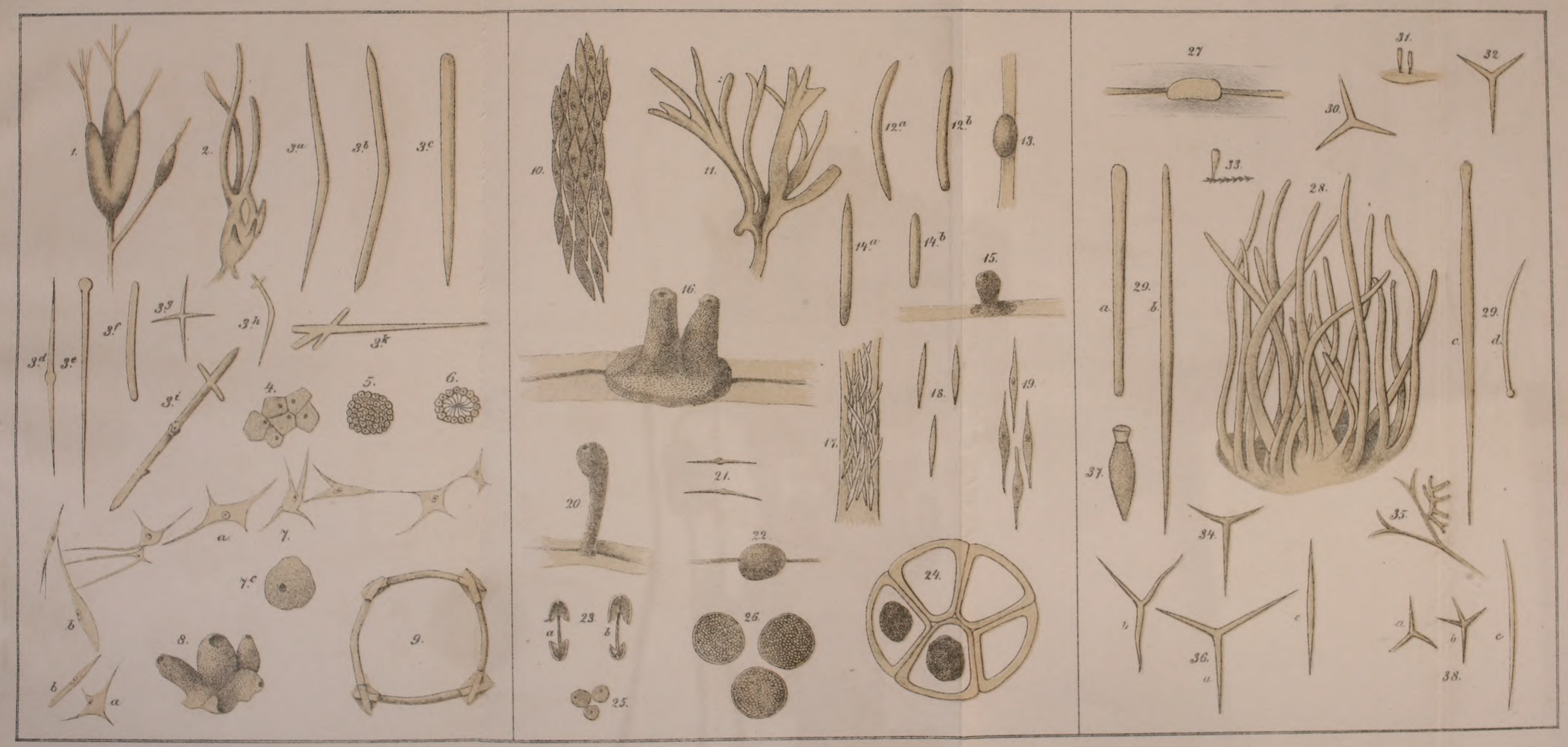


